Experimental validation of a distributed parameter piezoelectric bimorph cantilever energy harvester

This article has been downloaded from IOPscience. Please scroll down to see the full text article.

2010 Smart Mater. Struct. 19094008

(http://iopscience.iop.org/0964-1726/19/9/094008)

View the table of contents for this issue, or go to the journal homepage for more

Download details:

IP Address: 130.88.106.188

The article was downloaded on 12/01/2011 at 15:46

Please note that terms and conditions apply. 


\title{
Experimental validation of a distributed parameter piezoelectric bimorph cantilever energy harvester
}

\author{
S Rafique ${ }^{1}$ and $\mathbf{P}$ Bonello \\ School of Mechanical, Aerospace and Civil Engineering, University of Manchester, \\ Manchester M13 9PL, UK \\ E-mail: sajid.rafique@ postgrad.manchester.ac.uk
}

Received 19 January 2010, in final form 24 May 2010

Published 5 August 2010

Online at stacks.iop.org/SMS/19/094008

\begin{abstract}
Recent rapid advances in low-power portable electronic applications have motivated researchers and industry to explore schemes to embed an endless power supply mechanism within these systems. These self-charging embedded power supply systems convert ambient energy (vibration, solar, wind, etc) into electrical energy and subsequently provide power to these portable applications. Ambient vibration is one of the most promising sources of energy as it is abundantly present in indoor/outdoor systems. This paper discusses briefly the mathematical model of a bimorph piezoelectric cantilever beam with distributed inertia, and its experimental validation. Research on such a component typically included a tip mass, which reduced the influence of the distributed inertia of the beam and restricted effective operation to low frequencies. The present work excludes the tip mass and only the distributed mass of the harvester is considered. Due to the coupled electromechanical nature of piezoelectric materials, the effects of electrical coupling on the mechanical properties of the harvester are investigated, particularly the dependence of the induced additional stiffness and damping on the electrical load. Both the model and the experimental results show that the resonance frequency and the response amplitude of the harvester exhibit considerable shifts due to the electrical coupling. The experimental work uses both magnitude and Nyquist plots of the electromechanical frequency response functions to thoroughly validate the accuracy and applicability of the distributed parameter model at higher frequencies than previously considered.
\end{abstract}

(Some figures in this article are in colour only in the electronic version)

\section{Introduction}

Unused energy is ubiquitous in the environment in various forms. The potential ambient sources of energy include solar, thermal, wind and mechanical energy. Energy from these ambient sources is dissipated in the form of heat without being utilized for any useful purpose. The idea of extracting energy from these wasted or unused sources and then accumulating and storing it for a useful purpose is called energy harvesting. Among these sources, ambient vibration is one of the most promising sources of harvesting energy since it is present in most engineering systems and does not require any external electrical power source to initiate the

1 Author to whom any correspondence should be addressed. energy harvesting process. Piezoelectric materials have the ability to generate an electric potential when deformed due to vibrations (sensor effect). Conversely, they deform when subjected to an externally applied electric voltage (actuator effect). A range of devices that exploit this property have been investigated by many researchers working in the vibrationto-electricity energy harvesting field. The amount of power generated by vibration-to-electricity piezoelectric harvesters is quite low (in the range of micro to milliwatts $[1,2]$ ). However, this low amount is mainly due to the small size of these devices and their specific power can be quite high [1]. Moreover, over the past few years, rapid developments in low-power electronics and sensor technology have significantly reduced the power consumption within these systems. This low-power requirement has attracted researchers of diverse backgrounds 
to explore useful energy harvesting techniques that can provide continuous power to these portable systems.

Typically, portable electronic systems are powered by electrochemical batteries that need periodic recharging or replacement. Moreover, the progress in conventional battery technology has not been rapid enough to meet the continuous power requirements of these electronic systems [3]. Therefore, periodic battery recharging or replacement is required for the smooth functioning of these devices, which is not a suitable option in many cases, e.g., in remote areas where a regular electrical supply is not available. The idea of integration of a power harvesting system within these portable electronic devices and sensors will eliminate the need for periodic recharging or replacement of electrochemical batteries and will ensure uninterrupted functioning of these devices for a longer life span.

Preceding research work extensively investigated the use of piezoelectric materials in vibration-to-electricity energy harvesters [3, 4]. To investigate the practicability of the energy harvesting technique from vibrations, researchers not only presented different mathematical models [5-11] but also provided experimental results [1] to support their proposed models. Energy harvesting from ambient vibrations is a multidisciplinary field and involves extensive knowledge of the mechanics and electrical circuitry of the system. Researchers from different backgrounds are engaged extensively in the vibration-to-electricity energy harvesting field. Therefore, different oversimplified mathematical modeling assumptions were used in the literature to elucidate the vibrationto-electricity energy harvesting process. However, some major issues relating to the mathematical modeling of piezoelectric energy harvesters have been highlighted and addressed recently [12]. More generally, the research work in energy harvesting is focused on evaluating the performance of different piezoelectric materials [13], improving the geometrical and physical configuration of harvesters [2, 14] for maximizing output, improvements in mathematical modeling techniques [2, 5, 9, 12, 14], enhancing the capability of electrical circuitry to extract more power [15-17], and improving the power storage media [18, 19].

An energy harvester is typically a cantilevered beam with one or two piezoelectric layers (unimorph or bimorph respectively). This is attached at its base to a vibrating host structure. In practical implementations, a sophisticated electrical circuit, involving alternating-current-to-direct-current conversion, is connected to enable charging of a battery or storage capacitor. However, researchers typically consider a simple resistive load connected across the harvester to come up with mathematical models to predict the electrical output for a given base motion input [1]. The mathematical modeling used in energy harvesting literature includes lumped parameter single degree of freedom (SDOF) models [2, 20], the discrete parameter Rayleigh-Ritz method [9] and the distributed parameter modeling approach $[1,21]$. Although the SDOF approach provides preliminary understanding of an energy harvesting system, it is oversimplified and overlooks several important features such as the dynamic mode shapes and the accuracy of the strain distribution along the bender [21]. Therefore, a more detailed distributed parameter modeling approach is required to correctly model the behavior of the structure at any point along its length. Conversion of mechanical energy of the vibrating structure into electrical energy introduces additional damping [22] and affects other dynamic properties of the device, namely its effective stiffness, and consequently its resonance frequencies. As the value of the connected electrical load is increased, the backward piezoelectric coupling effect becomes prominent and not only attenuates the vibration amplitude but shifts the resonance frequency of the harvester during the energy harvesting process [21]. A closed-form distributed parameter (continuous) modeling approach for a base-excited clamped-free unimorph without any tip mass was introduced by Erturk and Inman [21]. This study was not experimentally validated. In a later paper [1], the same authors presented an experimentally validated model of a base-excited continuous bimorph with a lumped tip mass.

The present paper presents an experimentally validated distributed parameter model of a base-excited clamped-free bimorph without any lumped tip mass. Frequency response functions (FRFs) are derived for the electrical and mechanical outputs of a bimorph. As in the previous studies [1,21], the Euler-Bernoulli beam theory and the constitutive relations of piezoelectric materials are used. The novel aspects of this paper relative to $[1,21]$ are listed as follows:

- No tip mass is used in the present study. The authors consider this to be a more stringent validation of the distributed parameter piezoelectric beam model, since the presence of a tip mass reduces the influence of the distributed inertia of the beam and restricts effective operation to low frequencies (e.g. $45-50 \mathrm{~Hz}$ resonance in [1]). In the present case the resonance frequencies are in the range 120-130 Hz. This covers the higher end of the frequency range of application that most harvesters are designed for.

- The paper presents graphs showing the theoretical and experimental variation with electrical load of the resonance frequency, resonant voltage amplitude, resonant power and resonant deflection amplitude. These graphs give a deeper insight into the electromechanical interaction. Previous studies e.g. [1, 21] have only shown the variation with load of the voltage and power at two fixed excitation frequencies (equal to the short and open circuit resonances respectively).

- Nyquist plots of the FRFs are presented. FRFs are complex-valued functions. However, previous work focused only on the magnitude-frequency plots of the FRFs. The Nyquist plots are used for two purposes:

* A more thorough validation of the evolution of the FRFs as the electrical load is varied.

* The estimation of the mechanical damping.

The curve-fitting method used in [1] involved finding, by trial and error, the mechanical damping ratio that gave best agreement between the theoretical and experimental magnitude-frequency plots of the FRFs. As shall be seen in this paper, a method for mechanical damping estimation based on the Nyquist plot does not involve trial and error and is, in itself, a means for validating the model. 


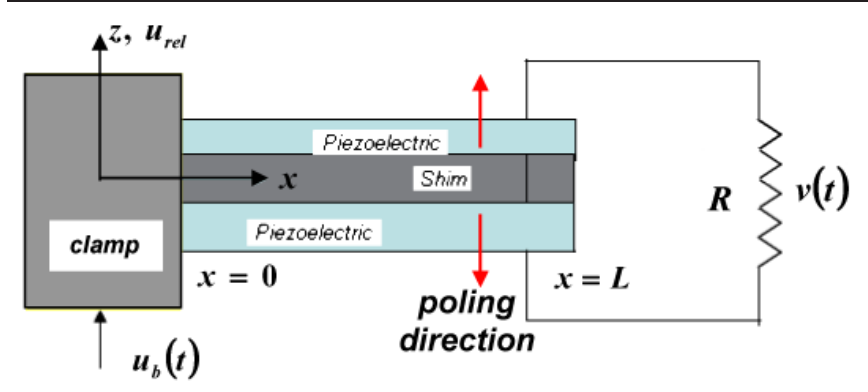

Figure 1. Cantilevered series-connected bimorph excited by base translation.

- The measured FRFs are obtained through the application of random excitation (band-limited white noise) rather than a sine sweep (used in [1]).

\section{Distributed parameter model of a bimorph}

In this section, a distributed parameter model of a piezoelectric bimorph beam without any lumped or tip mass is discussed. A bimorph consists of two piezoelectric layers bonded on the top and bottom surfaces of a central metallic shim, as shown in figure 1. Therefore, a bimorph can be electrically connected either in series or in parallel depending on the poling directions of the top and bottom piezoelectric layers. When the two piezoelectric layers are poled in the opposite direction then the harvester is connected in series, as shown in figure 1, where the opposite arrows on the top and bottom piezoelectric layers illustrate their opposite poling directions. When the two layers have the same poling direction then the harvester is connected in parallel. In series connectivity, a higher voltage and low current is achieved whereas a higher current and low voltage is attained in the parallel connection configuration. However, the net power output remains the same whether the device is connected in series or in parallel since it is the product of voltage and current [2]. The bimorph used in this work is connected in series, as shown in figure 1 . In the following sections the coupled electromechanical FRFs of the base-excited bimorph are derived for the case of harmonic excitation and no rotation at the clamp. The derivation follows that in [1] except that there is no attached lump mass at the tip. The main steps of the derivation are presented here for completeness. The applicability of the FRFs to random base excitation (not considered in [1]) is then discussed.

\subsection{Mechanical equation with electrical coupling}

In this section, a dynamic equation of motion for a bimorph beam in the mechanical domain with electrical back-coupling is obtained.

Let $\left(\sum Y I\right)$ be the bending stiffness of the composite beam (the summation sign refers to the fact that the cross section is made of more than one material), $m$ the distributed mass per unit length, $R$ the connected electrical load and $v(t)$ the voltage appearing across the resistor. Assuming that the rotation of the clamp is negligible, the absolute transverse displacement of the beam at a distance $x$ from the fixed end can be represented as [23]:

$$
u(x, t)=u_{\mathrm{b}}(t)+u_{\mathrm{rel}}(x, t)
$$

where $u_{\mathrm{b}}(t)$ is the absolute transverse displacement of the clamp and $u_{\text {rel }}(x, t)$ is the transverse displacement at distance $x$ relative to the moving base (i.e. the flexural displacement).

The governing equation of motion of an Euler-Bernoulli beam can be written as [21]:

$$
\begin{aligned}
& \frac{\partial^{2} M(x, t)}{\partial x^{2}}+\left(\sum c I\right) \frac{\partial^{5} u_{\text {rel }}(x, t)}{\partial x^{4} \partial t}+c_{\mathrm{a}} \frac{\partial u_{\text {rel }}(x, t)}{\partial t} \\
& +m \frac{\partial^{2} u_{\text {rel }}(x, t)}{\partial t^{2}}=-m \frac{\partial^{2} u_{\mathrm{b}}(x, t)}{\partial t^{2}}-c_{\mathrm{a}} \frac{\partial u_{\mathrm{b}}(x, t)}{\partial t}
\end{aligned}
$$

where $M$ is the internal elastic bending moment (excluding the distributed internal viscoelastic effect), $\left(\sum c I\right)$ is the equivalent viscoelastic damping term of the composite cross section, $c_{\mathrm{a}}$ is the ambient viscous damping coefficient per unit length and $m$ is the mass per unit length of the beam. It should be noted that both viscoelastic and ambient damping terms satisfy proportional damping criteria so it is mathematically convenient to use them in the modal analysis solution [21].

In equation (2), the relative transverse displacement with respect to the vibrating base $u_{\text {rel }}(x, t)$ can be written in modal coordinates using the modal expansion theorem [24]:

$$
u_{\mathrm{rel}}(x, t)=\sum_{r=1}^{\infty} \phi_{r}(x) \eta_{r}(t)
$$

where $\phi_{r}(x)$ are the mass-normalised eigenfunctions of the $r$ th mode of the cantilever beam and $\eta_{r}(t)$ the corresponding modal coordinates. The eigenfunctions $\phi_{r}(x)$ pertain to undamped, electrically uncoupled conditions and are given by [25]:

$$
\begin{aligned}
\phi_{r}(x) & =\sqrt{\frac{1}{m L}}\left[\cosh \frac{\lambda_{r}}{L} x-\cos \frac{\lambda_{r}}{L} x\right. \\
- & \left.\sigma_{r}\left(\sinh \frac{\lambda_{r}}{L} x-\sin \frac{\lambda_{r}}{L} x\right)\right]
\end{aligned}
$$

where $\sigma_{r}$ is

$$
\sigma_{r}=\frac{\left(\sinh \lambda_{r}-\sin \lambda_{r}\right)}{\left(\cosh \lambda_{r}+\cos \lambda_{r}\right)}
$$

$L$ is the length of the beam and the $\lambda_{r} \mathrm{~s}$ are dimensionless frequency parameters that can be determined by the following transcendental characteristic equation of a clamp-free beam:

$$
1+\cos \lambda_{r} \cosh \lambda_{r}=0 \text {. }
$$

The undamped natural frequency of the $r$ th mode of a uniformly distributed clamped-free beam can be written as:

$$
\omega_{r}=\lambda_{r}^{2} \sqrt{\frac{\left(\sum Y I\right)}{m L^{4}}} .
$$

The internal moment $M$ in equation (2) can be determined using the constitutive relations of the piezoelectric material [26]. Figure 2 shows the cross section of a bimorph beam 


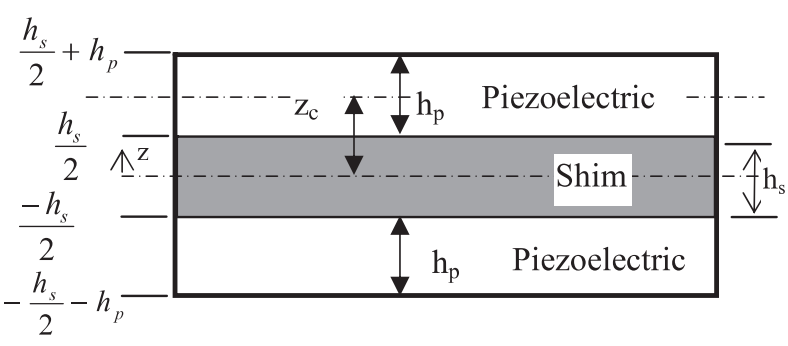

Figure 2. Cross section of a bimorph, distances positive upwards from the neutral/central axis.

having a metallic shim sandwiched between top and bottom piezoelectric layers.

Bending of the piezo layers produces a stress in the ' 1 ' direction which, in turn, induces a voltage in the ' 3 ' direction. Hence, the constitutive equations of the piezoelectric layers, can be written as [26]:

$$
\begin{aligned}
& D_{3}=d_{31} \sigma_{\mathrm{p}}+\varepsilon_{33}^{T} E_{3} \\
& \delta_{\mathrm{p}}=\sigma_{\mathrm{p}} / Y_{\mathrm{p}}+d_{31} E_{3}
\end{aligned}
$$

where $D_{3}$ is the electrical displacement (charge density) in the electric poling direction, $d_{31}$ is the piezoelectric strain coefficient, $\sigma_{\mathrm{p}}$ and $\delta_{\mathrm{p}}$ are the stress and strain in the piezoelectric layer, $Y_{\mathrm{p}}$ is Young's modulus of the piezoelectric material, $E_{3}$ is the electric field and $\varepsilon_{33}^{T}$ is the permittivity at constant stress. The stress in the central metallic layer (shim) can simply be expressed by Hooke's law as $\sigma_{\mathrm{s}}=Y_{\mathrm{s}} \delta_{\mathrm{s}}$ where $Y_{\mathrm{s}}$ is Young's modulus of elasticity for the shim and $\delta_{\mathrm{s}}, \sigma_{\mathrm{s}}$ are the axial strain and stress respectively.

During bending of the beam, the stresses in the top and bottom piezoelectric layers at a given section $x$ will be in opposite directions (one is in tension and the other is in compression) [1]. It is for this reason that, in the present case of series-connected piezo layers, the piezo layers have to be oppositely poled [27]. The internal moment produced by these opposite stresses in the top and bottom piezoelectric layers and the stress in the shim can then be written as:

$$
\begin{gathered}
M(x, t)=-\int_{\frac{h_{\mathrm{s}}}{2}}^{\frac{h_{\mathrm{s}}}{2}+h_{\mathrm{p}}} Y_{\mathrm{p}}\left(\delta-d_{31} E_{3}\right) b z \mathrm{~d} z-\int_{\frac{-h_{\mathrm{s}}}{2}}^{\frac{h_{\mathrm{s}}}{2}} Y_{\mathrm{s}} \delta b z \mathrm{~d} z \\
\quad-\int_{\frac{-h_{\mathrm{s}}}{2}-h_{\mathrm{p}}}^{\frac{-h_{\mathrm{s}}}{2}} Y_{\mathrm{p}}\left(\delta+d_{31} E_{3}\right) b z \mathrm{~d} z
\end{gathered}
$$

where $h_{\mathrm{p}}$ is the thicknesses of each piezoelectric layer and $h_{\mathrm{s}}$ is the thickness of the shim. $\delta$ is the generic strain in the beam at a distance $z$ from the neutral axis. Substituting for the strain $\delta=-z \partial^{2} u_{\text {rel }} / \partial x^{2}$ and the electric field $E_{3}=-v(t) /\left(2 h_{\mathrm{p}}\right)$ and performing the integrations in equation (10) will yield:

$$
M(x, t)=\left(\sum Y I\right) \frac{\partial^{2} u_{\text {rel }}(x, t)}{\partial^{2} x}+\Theta v(t)
$$

where $\left(\sum Y I\right)$ is calculated as:

$$
\left(\sum Y I\right)=b\left[\frac{Y_{\mathrm{s}} h_{\mathrm{s}}^{3}}{12}+Y_{\mathrm{p}}\left(\frac{h_{\mathrm{p}} h_{\mathrm{s}}^{2}}{2}+h_{\mathrm{p}}^{2} h_{\mathrm{s}}+\frac{2}{3} h_{\mathrm{p}}^{3}\right)\right]
$$

and $\Theta$ is the electrical coupling term in the internal bending moment (mechanical domain) expression and is determined as:

$$
\Theta=-\frac{d_{31} Y_{\mathrm{p}} b\left(h_{\mathrm{p}}+h_{\mathrm{s}}\right)}{2} .
$$

It should be noted that the piezoelectric coupling term, $\Theta v(t)$, in equation (11) is a function of time only, so it should be multiplied by $[H(x)-H(x-L)]$, where $H(x)$ is a Heaviside function [1], before substituting it into the equation of motion (equation (2)). The resulting equation of motion is then transformed from physical to modal coordinates by substituting for $u_{\text {rel }}$ from equation (3), and applying the orthogonality conditions of the eigenfunctions [21] will yield:

$$
\begin{aligned}
& \frac{\mathrm{d}^{2} \eta_{r}(t)}{\mathrm{d} t^{2}}+2 \xi_{r} \omega_{r} \frac{\mathrm{d} \eta_{r}(t)}{\mathrm{d} t}+\omega_{r}^{2} \eta_{r}(t)+\chi_{r} v(t) \\
& =-m \frac{\partial^{2} u_{\mathrm{b}}(x, t)}{\partial t^{2}} \int_{x=0}^{L} \phi_{r}(x) \mathrm{d} x
\end{aligned}
$$

where $\chi_{r}$ contains electrical and mechanical terms and can be written as:

$$
\chi_{r}=\left.\Theta \frac{\mathrm{d} \phi_{r}(x)}{\mathrm{d} x}\right|_{x=L} .
$$

It is noted that the excitation provided by the ambient damping (second term on the right-hand side of equation (2)) has been neglected, as in $[1,21]$.

\subsection{Electrical circuit equation with mechanical coupling}

In this section, the electrical circuit equation with mechanical back-coupling term will be obtained using the piezoelectric constitutive relations given in equations (8) and (9).

The total charge generated at the electrodes can be determined by integrating equation (8) over the whole area:

$$
q=\int_{x=0}^{L}\left(d_{31} Y_{\mathrm{p}} \bar{\delta}_{\mathrm{p}}+\varepsilon_{33}^{\mathrm{S}} E_{3}\right) b \mathrm{~d} x
$$

where $b$ is the width of the piezoelectric layer and $\bar{\delta}_{\mathrm{p}}$ is the bending strain along the middle surface of the upper piezo layer. The current through a load resistor $R$ is the time derivative of the charge:

$$
i(t)=\frac{v(t)}{R}=\frac{\mathrm{d}}{\mathrm{d} t}\left[\int_{x=0}^{L}\left(d_{31} Y_{\mathrm{p}} \bar{\delta}_{\mathrm{p}}+\varepsilon_{33}^{\mathrm{S}} E_{3}\right) b \mathrm{~d} x\right] .
$$

Substituting for the electric field $E_{3}=-v(t) /\left(2 h_{\mathrm{p}}\right)$ and the strain $\bar{\delta}_{\mathrm{p}}=-z_{\mathrm{c}} \partial^{2} u_{\text {rel }} / \partial x^{2}$, where $z_{\mathrm{c}}=\left(h_{\mathrm{p}}+h_{\mathrm{s}}\right) / 2$ (as in figure 2) in equation (17) will yield:

$$
\left(\frac{\varepsilon_{33}^{\mathrm{S}} b L}{2 h_{\mathrm{p}}}\right) \frac{\mathrm{d} v(t)}{\mathrm{d} t}+\frac{v(t)}{R}=-Y_{\mathrm{p}} d_{31} b z_{\mathrm{c}} \int_{x=0}^{L} \frac{\partial^{3} u_{\mathrm{rel}}(x, t)}{\partial x^{2} \partial t} \mathrm{~d} x .
$$

Substituting the value of $u_{\text {rel }}(x, t)$ from equation (3) into (18) then gives:

$$
\frac{C_{\mathrm{p}} \mathrm{d} v(t)}{2 \mathrm{~d} t}+\frac{v(t)}{R}=\sum_{r=1}^{\infty} \alpha_{r} \frac{\mathrm{d} \eta_{r}(t)}{\mathrm{d} t}
$$


where $\alpha_{r}$ is a constant and can be written as:

$\alpha_{r}=-Y_{\mathrm{p}} d_{31} b z_{\mathrm{c}} \int_{x=0}^{L} \frac{\mathrm{d}^{2} \phi_{r}(x)}{\mathrm{d} x^{2}} \mathrm{~d} x=-\left.Y_{\mathrm{p}} d_{31} b z_{\mathrm{c}} \frac{\mathrm{d} \phi_{r}(x)}{\mathrm{d} x}\right|_{x=L}$.

In equation (19), the capacitance $C_{\mathrm{p}}$ of the piezoelectric element can be defined as:

$$
C_{\mathrm{p}}=\frac{\varepsilon_{33}^{\mathrm{S}} b L}{h_{\mathrm{p}}}
$$

where $\varepsilon_{33}^{\mathrm{S}}$ is the permittivity at constant strain and can be written as $\varepsilon_{33}^{\mathrm{S}}=\varepsilon_{33}^{T}-d_{31}^{2} Y_{\mathrm{p}}$. It is stated in the literature that a piezoelectric can be represented as a current source in parallel with its internal capacitance. The components of the electrical circuit are the internal capacitance of the piezoelectric layers, the current source $i_{\mathrm{p}}(t)$ and the externally connected load resistor $R$ [1].

\subsection{Derivation of FRFs}

Assuming the base excitation $u_{\mathrm{b}}(t)$ is harmonic:

$$
u_{\mathrm{b}}=\operatorname{Re}\left\{U_{\mathrm{b}} \mathrm{e}^{\mathrm{j} \omega t}\right\}
$$

then all time-varying quantities are harmonic and can be expressed as:

$$
\begin{gathered}
v(t)=\operatorname{Re}\left\{V \mathrm{e}^{\mathrm{j} \omega t}\right\}, \\
u(x, t)=\operatorname{Re}\left\{U(x) \mathrm{e}^{\mathrm{j} \omega t}\right\}, \\
u_{\text {rel }}(x, t)=\operatorname{Re}\left\{U_{\text {rel }}(x) \mathrm{e}^{\mathrm{j} \omega t}\right\}, \\
\eta_{r}(t)=\operatorname{Re}\left\{\tilde{\eta}_{r} \mathrm{e}^{\mathrm{j} \omega t}\right\}
\end{gathered}
$$

where $U_{\mathrm{b}}, V, U(x), U_{\text {rel }}(x)$ and $\tilde{\eta}_{r}$ are the complex amplitudes of the respective quantities and $\omega$ is the excitation frequency in $\operatorname{rad~s}^{-1}$. Substituting for $u_{\mathrm{b}}(t), v(t)$ and $\eta_{r}(t)$ from equations $(22 a),(22 b),(22 e)$ into equation (14) and rearranging gives:

$$
\tilde{\eta}_{r}=\frac{\left(F_{r}-\chi_{r} V\right)}{\omega_{r}^{2}-\omega^{2}+\mathrm{j} 2 \xi_{r} \omega_{r} \omega}
$$

where

$$
\begin{gathered}
F_{r}=m \omega^{2} \gamma_{r}^{u} U_{\mathrm{b}}, \\
\gamma_{r}^{u}=\int_{x=0}^{L} \phi_{r}(x) \mathrm{d} x=\frac{2 \sigma_{r}}{\lambda_{r}} \sqrt{\frac{L}{m}} .
\end{gathered}
$$

Substituting for $v(t)$ and $\eta_{r}(t)$ from equations (22a) and (22e) into (19), and then substituting for $\tilde{\eta}_{r}$ from equation (23) and rearranging, gives the voltage FRF, denoted by $\breve{V}(\omega)$, as:

$$
\breve{V}(\omega)=\frac{V}{-\omega^{2} U_{\mathrm{b}}}=\frac{\sum_{r=1}^{\infty} \frac{-\mathrm{j} m \omega \gamma_{r}^{u} \alpha_{r}}{\omega_{r}^{2}-\omega^{2}+\mathrm{j} 2 \xi_{r} \omega_{r} \omega}}{\left(\frac{1}{R}+\mathrm{j} \omega \frac{C_{\mathrm{p}}}{2}\right)+\sum_{r=1}^{\infty} \frac{\mathrm{j} \omega \alpha_{r} \chi_{r}}{\omega_{r}^{2}-\omega^{2}+\mathrm{j} 2 \xi_{r} \omega_{r} \omega}} .
$$

The voltage FRF $\breve{V}(\omega)$ is seen to be the complex amplitude of the voltage divided by the complex amplitude of the base acceleration $\ddot{u}_{\mathrm{b}}$. The 'current FRF' is the complex amplitude of the current divided by the complex amplitude of $\ddot{u}_{\mathrm{b}}$. Hence, it is simply the voltage FRF divided by the resistance $R$. The instantaneous power is $v^{2} / R$. The peak power is therefore $|V|^{2} / R$ [1]. Hence, a measure of the power generated is obtained by taking the square of the modulus of the voltage FRF and dividing it by $R$. This quantity is defined as the 'power FRF'.

Substituting for $V$ from equation (25) into (23) and using equation (3) will yield the relative tip response FRF as:

$$
\begin{aligned}
\beta(\omega) & =\frac{U_{\mathrm{rel}}(L)}{-\omega^{2} U_{\mathrm{b}}} \\
= & -\sum_{r=1}^{\infty}\left\{\begin{array}{l}
\sum_{r=1}^{\infty} \frac{\mathrm{j} \omega \gamma_{r}^{u} \alpha_{r}}{\omega_{r}^{2}-\omega^{2}+\mathrm{j} 2 \xi_{r} \omega_{r} \omega} \\
\left.\times \frac{m \phi_{r}(L)}{\left(\frac{1}{R}+\mathrm{j} \omega \frac{C_{\mathrm{p}}}{2}\right)+\sum_{r=1}^{\infty} \frac{\mathrm{j} \omega \alpha_{r} \chi_{r}}{\omega_{r}^{2}-\omega^{2}+\mathrm{j} 2 \xi_{r} \omega_{r} \omega}}\right\}
\end{array}\right.
\end{aligned}
$$

where $U_{\text {rel }}(L)$ is the complex amplitude of the relative tip displacement (equation $(22 d)$ ). In practice, a laser sensor is used to measure the tip vibration, and this measures the absolute displacement or velocity. From equation (1), the absolute and relative tip displacement FRFs are related by the following equation:

$$
\beta_{\mathrm{abs}}(\omega)=-\frac{1}{\omega^{2}}+\beta(\omega)
$$

2.3.1. Reduced single-mode frequency response (FRFs) functions. In most of the energy harvesting research, only the fundamental or the first mode of the harvester is considered. The FRFs obtained in the last sections includes contributions of any number of modes. However, these FRFs can be reduced to a single-mode (first mode or for any mode $r$ ). The single-mode FRFs can be obtained by evaluating equations (25) and (26) for the $r$ th mode only and can be written as:

$$
\begin{aligned}
\left.\breve{V}(\omega)\right|_{r} & =\frac{-\mathrm{j} 2 \omega m R \gamma_{r}^{u} \alpha_{r}}{\left(2+\mathrm{j} \omega C_{\mathrm{p}} R\right)\left(\omega_{r}^{2}-\omega^{2}+\mathrm{j} 2 \xi_{r} \omega_{r} \omega\right)+\left(\mathrm{j} 2 \omega R \alpha_{r} \chi_{r}\right)} \\
\left.\beta(\omega)\right|_{r} & =\frac{-\left(2+\mathrm{j} \omega C_{\mathrm{p}} R\right) m \gamma_{r}^{u} \phi_{r}(L)}{\left(2+\mathrm{j} \omega C_{\mathrm{p}} R\right)\left(\omega_{r}^{2}-\omega^{2}+\mathrm{j} 2 \xi_{r} \omega_{r} \omega\right)+\left(\mathrm{j} 2 \omega R \alpha_{r} \chi_{r}\right)}
\end{aligned}
$$

The subscripts ' $r$ ' on the left-hand side of equations (28) and (29) indicate that the FRFs are single-mode approximations centered around the frequency $\omega_{r}$

\subsection{Application to non-harmonic base excitation}

The FRF expressions derived above relate, for harmonic excitation, the complex amplitude of the output (voltage or tip displacement) with the complex amplitude of the input (base acceleration). Following standard signal processing theory [28], since the system is linear, the same FRF expressions on the right-hand side of equations (25), (26), (28) and (29) should also be valid for non-harmonic excitation. In the case of a deterministic signal $u_{\mathrm{b}}$, the FRF expressions would define the ratio of the Fourier transforms of the output and input signals [28]. In the case of a non-deterministic (random i.e. broadband) signal $u_{\mathrm{b}}$, the FRFs would define the 
(a)
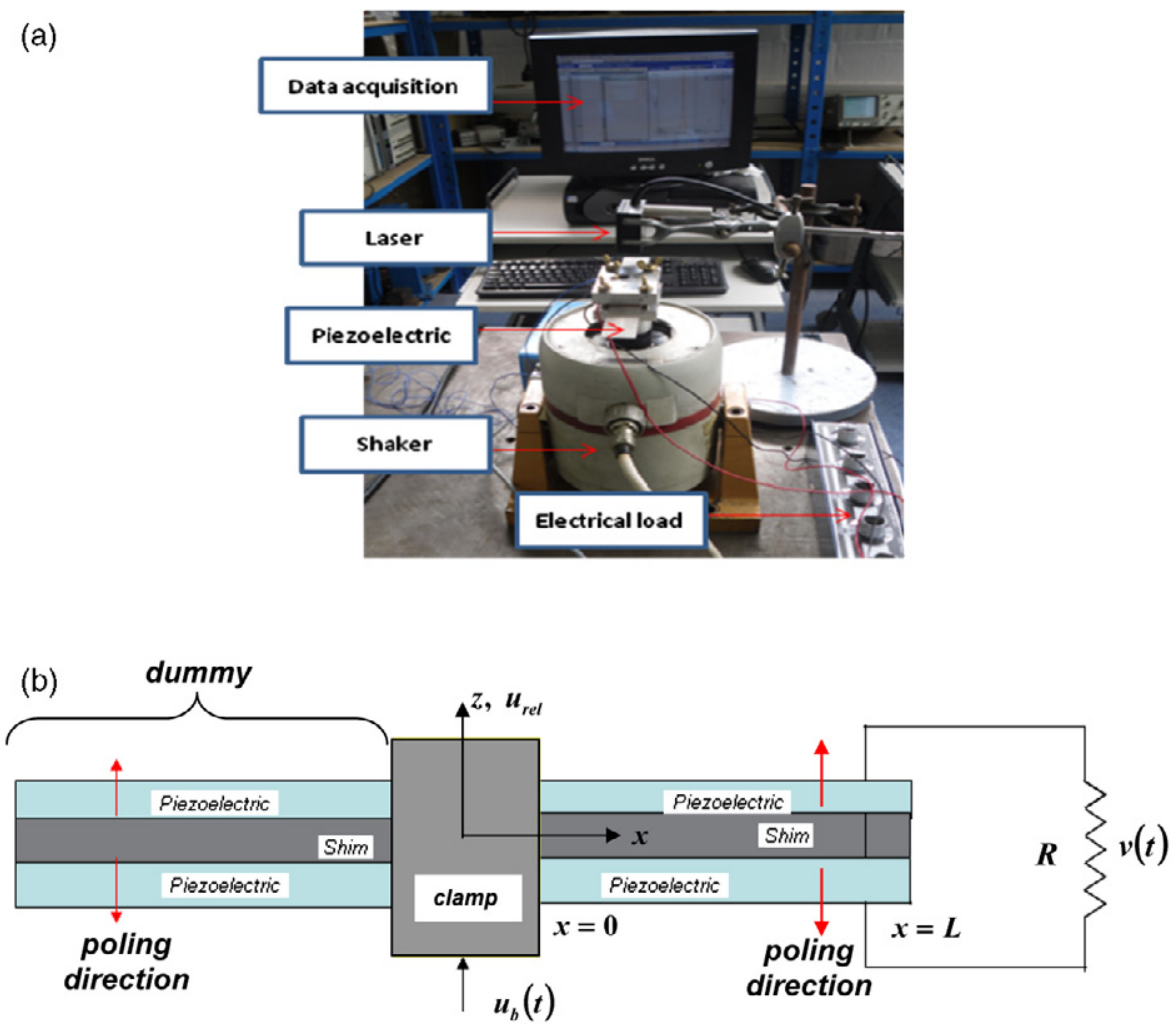

Figure 3. (a) Experimental setup. (b) Double cantilever arrangement to eliminate rotational effects at the clamp.

ratio of the cross-spectral density to the input power spectral density [28]. For example, the voltage FRF expression on the right-hand side of equation (25) or (28) would be equal to:

$$
G_{\ddot{u}_{\mathrm{b}} v}(\omega) / G_{\ddot{u}_{\mathrm{b}} \ddot{u}_{\mathrm{b}}}(\omega)
$$

where $G_{\ddot{u}_{\mathrm{b}} v}(\omega)$ is the cross-spectral density function relating signals $\ddot{u}_{\mathrm{b}}$ and $v$ and $G_{\ddot{u}_{\mathrm{b}}} \ddot{u}_{\mathrm{b}}(\omega)$ is the power spectral density function of $\ddot{u}_{\mathrm{b}}$.

\section{Experimentally validated study}

The above illustrated distributed parameter model of a bimorph was experimentally tested and the measured results were compared with those from the model. The experimental setup used is shown in figure 3(a). A bimorph with connected resistor was clamped at one end and the clamp mounted on an electrodynamic shaker. Rotational effects at the clamp were minimized by attaching an identical dummy bimorph at the other side of the clamp, as shown in figure 3(b). If a single cantilever is used, as in previous energy harvesting work [1], then the dynamic bending moment at the root of the cantilever tends to rotate the clamp. This tendency has to be resisted by an equal and opposite external moment applied by the shaker on the clamp. The reaction moment on the shaker potentially results in slight rocking of the shaker armature and clamp. The use of two symmetric cantilevers eliminates the moment on the shaker since the dynamic bending moments at the roots of the two cantilevers cancel each other out. It is common practice to implement a base-excited, no-baserotation cantilever as a symmetric double cantilever, as can be seen from references $[29,30]$.
Table 1. Properties of the bimorph harvester.

\begin{tabular}{|c|c|c|}
\hline Property & Units & Value \\
\hline Length of the beam and substrate & $\mathrm{mm}$ & 60 \\
\hline Width of the beam and substrate, $b$ & $\mathrm{~mm}$ & 25 \\
\hline $\begin{array}{l}\text { Thickness of each piezoelectric, } h_{\mathrm{p}} \\
\text { (upper and lower layers) }\end{array}$ & $\mathrm{mm}$ & 0.267 \\
\hline Thickness of the substrate, $h_{\mathrm{s}}$ & $\mathrm{mm}$ & 0.3 \\
\hline Young's modulus of the piezoelectric, $Y_{\mathrm{p}}$ & $\mathrm{GPa}$ & 62 \\
\hline Young's modulus of the substrate & $\mathrm{GPa}$ & 72 \\
\hline Density of the piezoelectric & $\mathrm{kg} \mathrm{m}^{-3}$ & 7800 \\
\hline Density of the substrate & $\mathrm{kg} \mathrm{m}^{-3}$ & 2700 \\
\hline Piezoelectric constant, $d_{31}$ & $\mathrm{pm} \mathrm{V}-1$ & -320 \\
\hline $\begin{array}{l}\text { Relative dielectric constant (at constant } \\
\text { stress) }\end{array}$ & & 3800 \\
\hline
\end{tabular}

The bimorphs were manufactured by Piezo Systems Inc. and each one was made up of two PZT-5H4E layers bonded on the top and bottom surfaces of an aluminum shim. As mentioned in the previous section, each bimorph was series-connected (figure 1) and its geometric, material and electromechanical properties, as provided by the manufacturer, are given in table 1. An accelerometer (PCB 352C22, sensitivity $9.08 \mathrm{mV} \mathrm{g}^{-1}$, resolution $0.002 \mathrm{~g} \mathrm{rms}$ ) was used to measure the base (clamp) acceleration $\ddot{u}_{0}$ and a laser sensor (MEL M5L/4-10B24NK, sensitivity $0.54 \mathrm{~V} \mathrm{~mm}^{-1}$, resolution $0.0005 \mathrm{~mm}$ ) was used to measure the absolute tip displacement $u(L, t)$.

The electrical load was purely resistive and was manually controlled through a resistor box. A PC-controlled dataacquisition system (LMS Scadas 5 with LMS Test.Lab Rev 

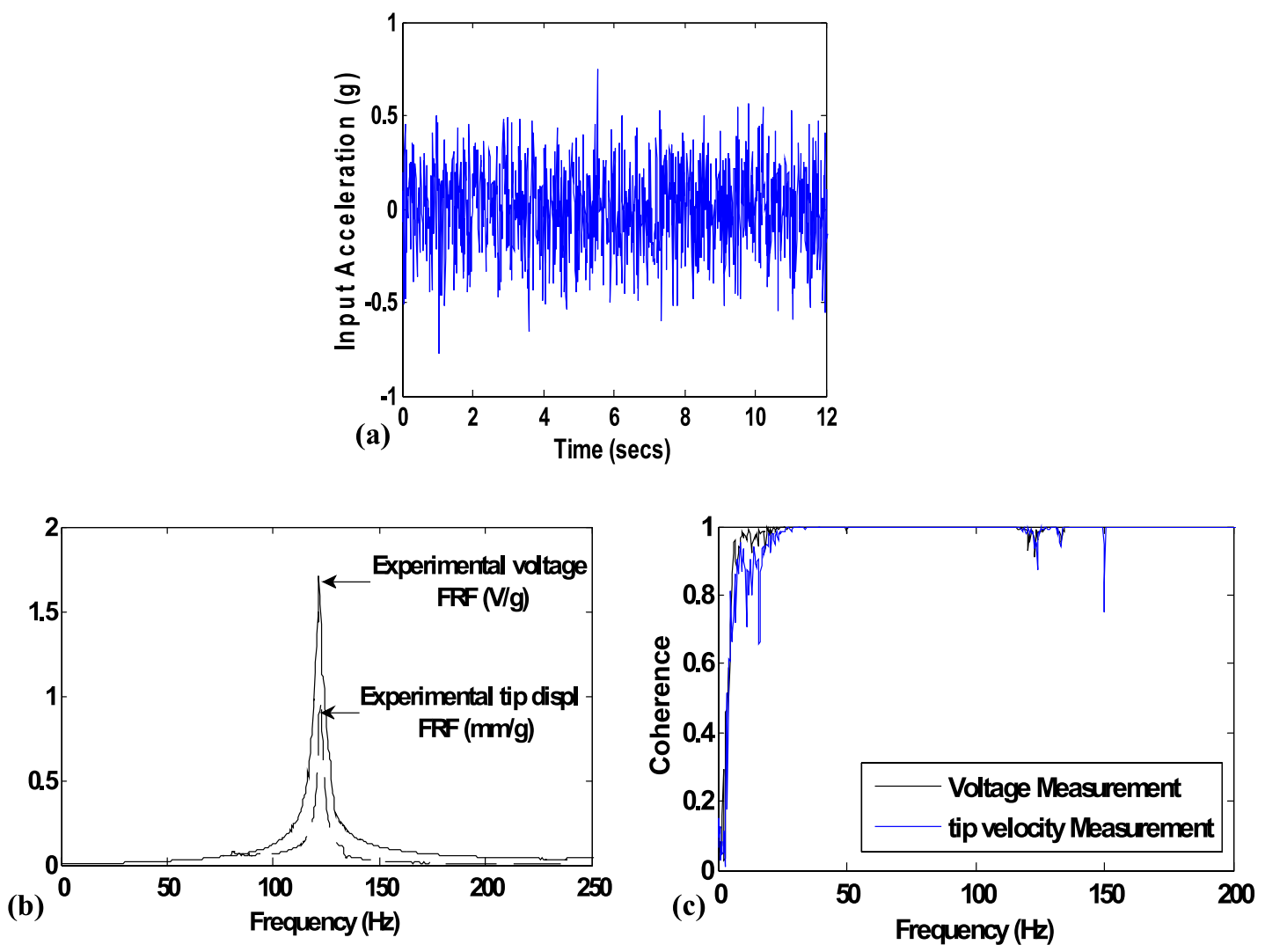

Figure 4. (a) Input acceleration at the base $\ddot{u}_{\mathrm{b}}$; (b) experimental voltage and tip velocity FRFs at $1 \mathrm{k} \Omega$ and (c) their coherence functions.

7A software) was used to generate an excitation signal to the shaker and to acquire the signals and calculate the FRFs.

The test device was excited by a random signal (bandlimited white noise) with a frequency spectrum of $0-320 \mathrm{~Hz}$ (i.e. containing the first undamped electrically uncoupled natural frequency $\omega_{1}$ ). The data-acquisition software computed the FRFs by calculating the cross-spectral densities and power spectral densities of the acquired signals, as discussed in section 2.4. Ten different electrical loads of $100 \Omega, 1 \mathrm{k} \Omega, 5 \mathrm{k} \Omega$, $10 \mathrm{k} \Omega, 15 \mathrm{k} \Omega, 25 \mathrm{k} \Omega, 50 \mathrm{k} \Omega, 100 \mathrm{k} \Omega, 500 \mathrm{k} \Omega$ and $1000 \mathrm{k} \Omega$ were used. The resonance frequency of the fundamental mode of the bender was measured at $121.7 \mathrm{~Hz}$ for a low value of $R=100 \Omega$. There was no observable shift in this frequency when $R$ was raised to the next higher load of $1 \mathrm{k} \Omega$. Hence, the measured value of the short circuit (i.e. electrically uncoupled) fundamental resonance (i.e. $\omega_{1}$ ) was $121.7 \mathrm{~Hz}$. This value is quite close to the theoretical value for $\omega_{1}(121.1 \mathrm{~Hz})$ calculated by equation (7). For all resistance values, the peak value of the random signal $\ddot{u}_{\mathrm{b}}$ did not exceed $1 \mathrm{~g}$ (see figure 4(a)). Operation in the linear regime was ascertained by monitoring the coherence functions relating $v$ with $\ddot{u}_{\mathrm{b}}$ and $u(L, t)$ with $\ddot{u}_{\mathrm{b}}$. For a linear system and in the absence of measurement noise, these coherence functions should be equal to unity over the frequency range of interest [28]. The experimental voltage FRF and tip velocity FRF at a load of $1 \mathrm{k} \Omega$ are shown in figure 4(b) along with the corresponding coherences in figure 4(c). It can be seen that the coherence is reasonable and approximately unity around the first resonance frequency.

\subsection{Estimation of the mechanical damping}

For $R$ small but not zero, the single-mode FRF expression of equations (28) and (29) can be approximately expressed as:

$$
\begin{aligned}
\left.\breve{V}(\omega)\right|_{r} & \approx \frac{-\mathrm{j} \omega m R \gamma_{r}^{u} \alpha_{r}}{\omega_{r}^{2}-\omega^{2}+\mathrm{j} 2\left\{\xi_{r}+\frac{R \alpha_{r} \chi_{r}}{2 \omega_{r}}\right\} \omega_{r} \omega}, \\
\left.\mathrm{j} \omega \beta(\omega)\right|_{r} & \approx \frac{-\mathrm{j} \omega m \gamma_{r}^{u} \phi_{r}(L)}{\omega_{r}^{2}-\omega^{2}+\mathrm{j} 2\left\{\xi_{r}+\frac{R \alpha_{r} \chi_{r}}{2 \omega_{r}}\right\} \omega_{r} \omega} .
\end{aligned}
$$

It is noted that the approximate expression for $\left.\beta(\omega)\right|_{r}$ has been multiplied by $\mathrm{j} \omega$ to obtain an expression that is of similar form to $\left.\breve{V}(\omega)\right|_{r}$. j $\left.\omega \beta(\omega)\right|_{r}$ is the single-mode expression for the relative tip velocity FRF (relative tip velocity amplitude per unit base acceleration amplitude). From modal testing theory [31], expressions of the form of $\left.\breve{V}(\omega)\right|_{r},\left.\mathrm{j} \omega \beta(\omega)\right|_{r}$ in equations (31) and (32) yield a circle passing through the origin with a diameter along the real axis when plotted as a Nyquist plot (i.e. real part versus imaginary part). This observation is approximately validated by experimental Nyquist plots of the voltage FRF and the relative tip velocity FRF for $1 \mathrm{k} \Omega$ over the frequency range $80-160 \mathrm{~Hz}$ (see figure 5), where the circles shown were fitted through the experimental data points using a least-squares fit. It is noted that the measured relative tip velocity FRF $\mathrm{j} \omega \beta(\omega)$ was obtained from the measured absolute tip displacement FRF $\beta(\omega)$ using the relation of equation (27).

From equations (31) and (32), it is evident that the equivalent electromechanical modal damping ratio $\hat{\xi}_{r}$, is given 

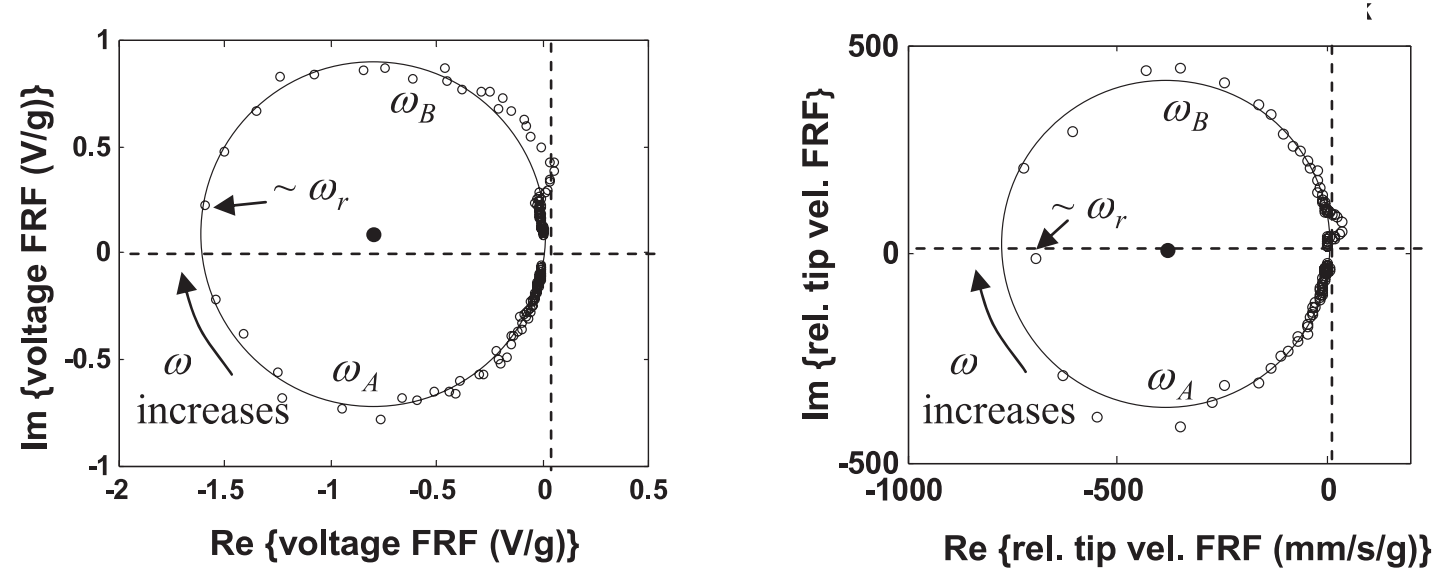

Figure 5. Experimental Nyquist plots at $1 \mathrm{k} \Omega$ of (a) voltage FRF and (b) relative tip velocity FRF (including circle-fits through data points).

by

$$
\hat{\xi}_{r}=\xi_{r}+\frac{R \alpha_{r} \chi_{r}}{2 \omega_{r}}
$$

From equation (31) or (32) and following a procedure similar to that in [31], it can be shown that $\hat{\xi}_{r}$ can be determined by the half-power-point formula:

$$
\hat{\xi}_{r}=\frac{\omega_{B}-\omega_{A}}{2 \omega_{r}} .
$$

According to equations (31) and (32), the condition $\omega=$ $\omega_{r}$ corresponds to the intersection with the real axis. Also, according to modal testing theory [31], for FRFs of the form of equations (31) and (32), the condition $\omega=\omega_{r}$ approximately corresponds to the location of maximal spacing between consecutive data points of the experimental Nyquist plot. These features are approximately validated in figure 5 . The locations of the half-power-point frequencies $\omega_{A, B}$ are displaced by $90^{\circ}$ on either side of the location of $\omega_{r}$. The frequencies in equation (34) can be located using the circlefit through the measured Nyquist plots in figure 5. As an alternative to the circle-fit (Nyquist plot) method, the frequencies in equation (34) could also be located using the magnitude-frequency plots of the voltage FRF or flexural tip velocity FRF ('peak amplitude method' [31]), figure 6.

The estimates for $\hat{\xi}_{1}$ obtained using both types of method (circle-fit, peak amplitude) for each type of FRF (voltage, flexural tip velocity FRF) are shown in table 2(a). For this particular case, the correlation between all estimates was found to be good. However, in general, one expects the circle-fit method to be more reliable since the peak amplitude method is highly sensitive to the frequency resolution. It is also noted that the values of $R$ significantly smaller than $1 \mathrm{k} \Omega$ resulted in considerable noise contamination in the voltage FRF (since the numerator of equation (31) is directly proportional to $R$ ). On the other hand, the tip velocity FRF could be used under pure short circuit conditions, as evident from equation (31). However, this FRF was potentially susceptible to errors introduced by the vibration of the laser head. Having determined the equivalent electromechanical

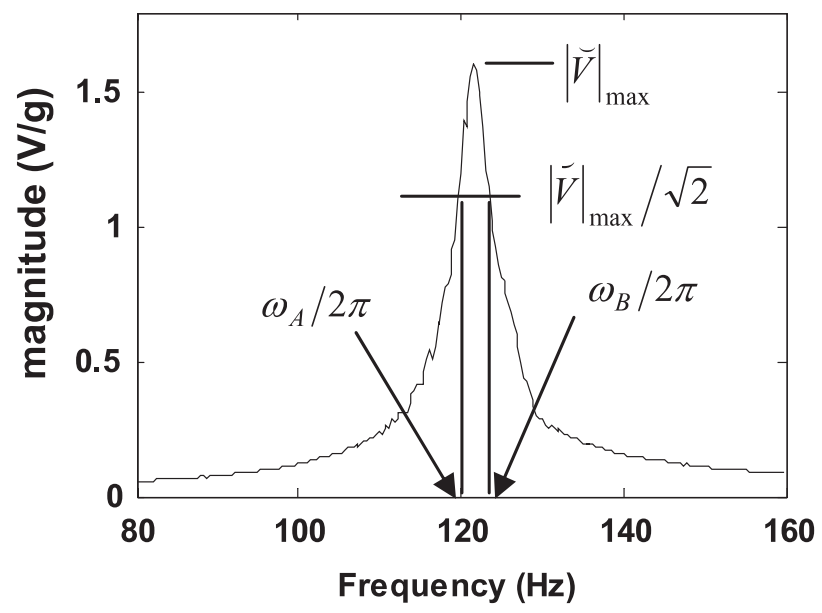

Figure 6. Peak amplitude method applied to voltage FRF at $1 \mathrm{k} \Omega$.

modal damping ratio $\hat{\xi}_{1}$, the mechanical modal damping ratio $\xi_{1}$ was determined from equation (33), since all parameters in the second term of this equation were quantifiable. The estimates for $\xi_{1}$ are shown in table 2(b). The average of the damping ratio values in table 2(b) was used for $\xi_{1}$ in the theoretical predictions of the following sections.

It is noted that in [1], $\omega_{r}$ was determined as the resonance at low resistance. $\xi_{r}$ was then determined in [1] by trial and error by fitting the theoretical magnitude-frequency plots of equations (28) or (29) to the experimental data. It is clear that the Nyquist plot method, presented here, not only avoids trial and error, but also provides a deeper insight and is itself a means for validating the theory.

\subsection{Comparison of theoretical and experimental FRFs}

This section analyses the electrical and mechanical FRFs for different resistances in the range $10^{2} \Omega$ ('short' circuit) to $10^{6} \Omega$ ('open' circuit). The resonance frequency at a given resistance is the frequency at which the magnitude of an FRF is greatest. It was observed that, for a given resistance, the peak 
Table 2. Estimates of modal damping ratio.

\begin{tabular}{llll}
\hline (a) equivalent electromechanical modal damping ratio $\hat{\xi}_{1}$ \\
\hline & $\begin{array}{l}\text { Load } R \\
(\mathrm{k} \Omega)\end{array}$ & $\begin{array}{l}\text { Circle-fit } \\
\text { method } \hat{\xi}_{1}(\%)\end{array}$ & $\begin{array}{l}\text { Peak amplitude } \\
\text { method } \hat{\xi}_{1}(\%)\end{array}$ \\
\hline $\begin{array}{l}\text { Relative tip } \\
\text { velocity FRF } \\
\text { Voltage FRF }\end{array}$ & 1 & 1.3 & 1.33 \\
\hline $\begin{array}{l}\text { (b) mechanical modal damping ratio } \xi_{1} \\
\text { Load } R\end{array}$ & $\begin{array}{l}\text { Circle-fit } \\
\text { method } \xi_{1}(\%)\end{array}$ & $\begin{array}{l}\text { Peak amplitude } \\
\text { method } \xi_{1}(\%)\end{array}$ \\
$\begin{array}{llll}\text { FRF } & 1 & 1.45\end{array}$ \\
\hline $\begin{array}{l}\text { Relative tip } \\
\text { velocity FRF }\end{array}$ & 1 & 1.0 & 1.03 \\
\begin{tabular}{l} 
Voltage FRF \\
\hline
\end{tabular} & 1 & 1.15 & 1.18 \\
\hline
\end{tabular}

magnitudes of all electrical and mechanical FRFs occurred at virtually the same frequency. This is in line with the model, as will be formally shown in the following sections. As mentioned previously, it was observed that, for resistances of up to $1 \mathrm{k} \Omega$, the value of the measured resonance frequency $(121.7 \mathrm{~Hz})$ was virtually unaffected by the resistance value. The reason for this is that, for small non-zero resistance, the electrical effect is equivalent to a viscous damper proportional to $R$, as evident from equation (32). For higher resistance values (up to $500 \mathrm{k} \Omega$ ) the measured resonance frequency increased with resistance, as will be shown in the following sections. It is noted that all simulations were obtained using the single-mode approximations in equations (28) and (29) with $r=1$. It should be noted that these equations were multiplied by $g\left(9.81 \mathrm{~m} \mathrm{~s}^{-2}\right)$ since the measured base acceleration was in $g \mathrm{~s}$ of acceleration.

3.2.1. Magnitude plots of voltage FRFs. Figure 7 shows the magnitude-frequency plots of the measured and predicted voltage FRFs. The increase in resonance frequency with load is clearly evident. The measured resonance frequency at $500 \mathrm{k} \Omega$ was $128.6 \mathrm{~Hz}$. This value was almost unaffected by increasing the resistance to $1 \mathrm{M} \Omega$. Hence, this frequency can be regarded as the 'open circuit' frequency. The theoretical open circuit frequency of $128.4 \mathrm{~Hz}$ correlates reasonably well with the corresponding measured value.

The theoretical variation of the resonance frequency with resistance can be obtained by taking the modulus of $\left.\bar{V}(\omega)\right|_{r}$ in equation (28), differentiating this with respect to $\omega$, and equating the result to 0 . The resulting equation will be the following cubic in $\omega^{2}$ :

$$
A_{\mathrm{a}}\left(\omega^{2}\right)^{3}+A_{\mathrm{b}}\left(\omega^{2}\right)^{2}+A_{\mathrm{c}} \omega^{2}+A_{d}=0
$$

where

$$
\begin{gathered}
A_{\mathrm{a}}=2 K_{\mathrm{a}}^{2}, \\
A_{\mathrm{b}}=4+K_{\mathrm{a}}^{2} K_{\mathrm{b}}^{2}-2 \omega_{r}^{2} K_{\mathrm{a}}^{2}-2 K_{\mathrm{a}} K_{\mathrm{c}}, \\
A_{\mathrm{c}}=0, \quad A_{d}=-4 \omega_{r}^{4} \\
K_{\mathrm{a}}=R C_{\mathrm{p}},
\end{gathered}
$$

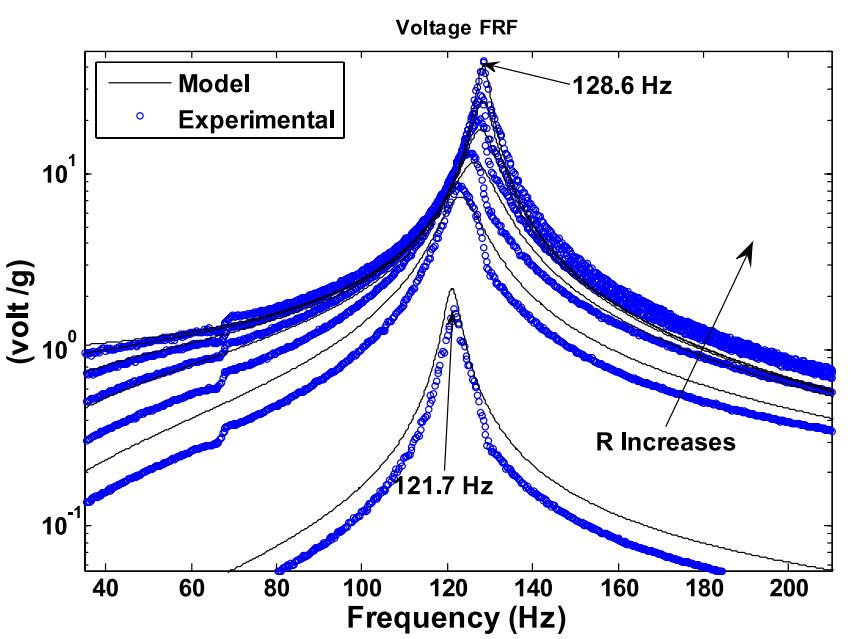

Figure 7. Voltage FRFs at six different loads $(1,10,25,50,100$, $500 \mathrm{k} \Omega$ ).

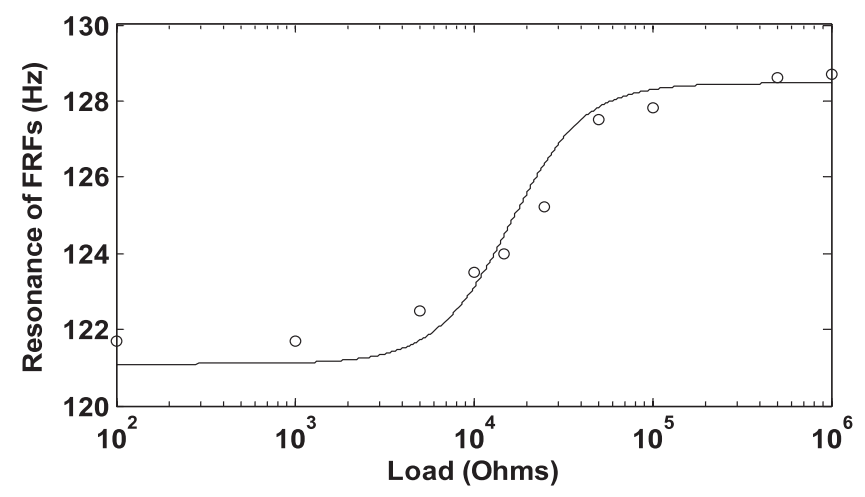

Figure 8. Variation of resonance frequency with electrical load (theoretical with experimental data points).

$$
\begin{gathered}
K_{\mathrm{b}}=2 \xi_{r} \omega_{r}, \\
K_{\mathrm{c}}=2 R \alpha_{r} \chi_{r} .
\end{gathered}
$$

The resonance frequency at given $R$ is then given by the positive real value of $\omega$ that satisfies the above equation. Solving the above equation for $r=1$ (fundamental mode) over a range of $R$ gives the curve in figure 8. It is seen that, at low resistances, the slope of the curve is horizontal. This is consistent with the observation that the rate of change of resonance frequency with $R$ is negligible for small $R$ due to the fact that, at such low resistances the electrical effect is only equivalent to viscous damping (equation (31)).

Figure 7 shows that the magnitude of the voltage FRF at resonance increases monotonically with the electrical load. These observations are illustrated in figure 9, where the magnitude of the voltage FRF at resonance (i.e. resonant voltage amplitude) is plotted as a function of load. The theoretical curve is obtained by evaluating the modulus of $\left.\breve{V}(\omega)\right|_{r}$ at the theoretical resonance frequencies of figure 8 .

Figure 7 shows that the magnitude of the voltage FRF at a fixed frequency also increases monotonically with the electrical load. This can be seen in figure 10, which illustrates the variation with electrical load of the value of the magnitude 


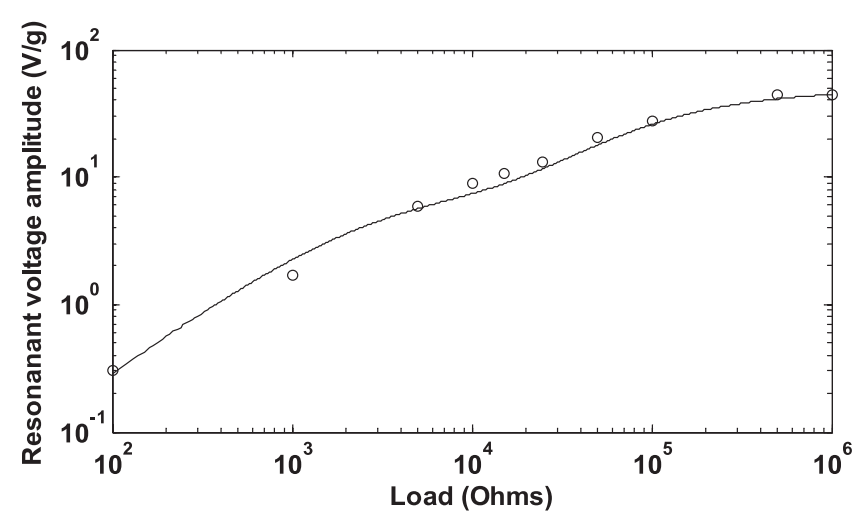

Figure 9. Variation of resonant voltage amplitude with electrical load (theoretical with experimental data points).

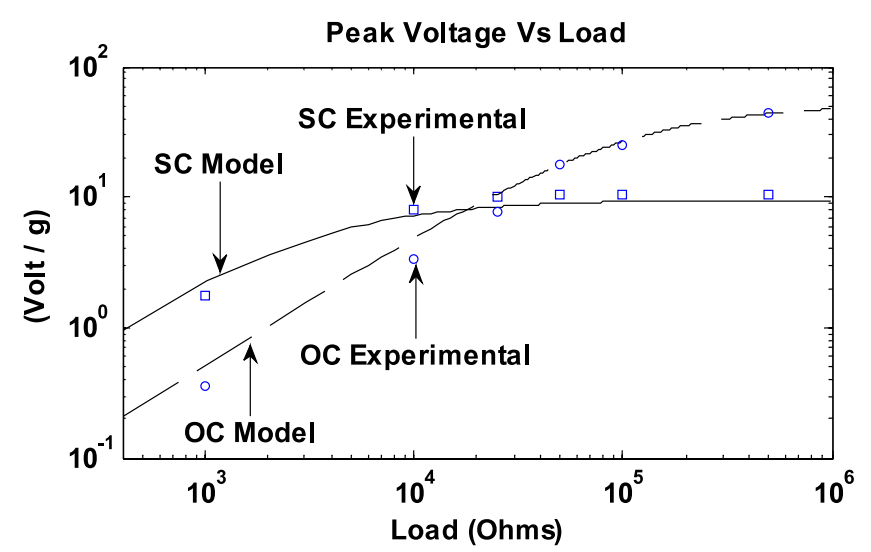

Figure 10. Voltage amplitude at short circuit and open circuit frequencies.

of the voltage FRF at the short circuit and open circuit frequencies. It shows that the voltage output at these two frequencies increases at a similar rate, but at low loads the output is higher for the short circuit frequency since the system is close to short circuit conditions [21].

The two curves intersect at a certain value, and at higher loads the voltage at the open circuit frequency becomes higher than that at the short circuit frequency since the system gets closer to open circuit conditions.

3.2.2. Magnitude plots of current FRFs. The current FRFs also showed a monotonic behavior with increase in load but in the reverse manner to the voltage. As the load was increased the amplitude of the current FRF was decreased. In figure 11, predicted and measured FRFs are compared for different electrical loads ranging from 1 to $500 \mathrm{k} \Omega$.

Figure 12 shows the magnitude of the current FRF at resonance (i.e. resonant current amplitude) plotted as a function of load. The behavior with increasing load is seen to be the reverse of that of figure 9 .

Figure 13 illustrates the variation with electrical load of the value of the magnitude of the current FRF at the short circuit and open circuit frequencies. The behavior with increasing load is again seen to be the reverse of that in figure 10 .

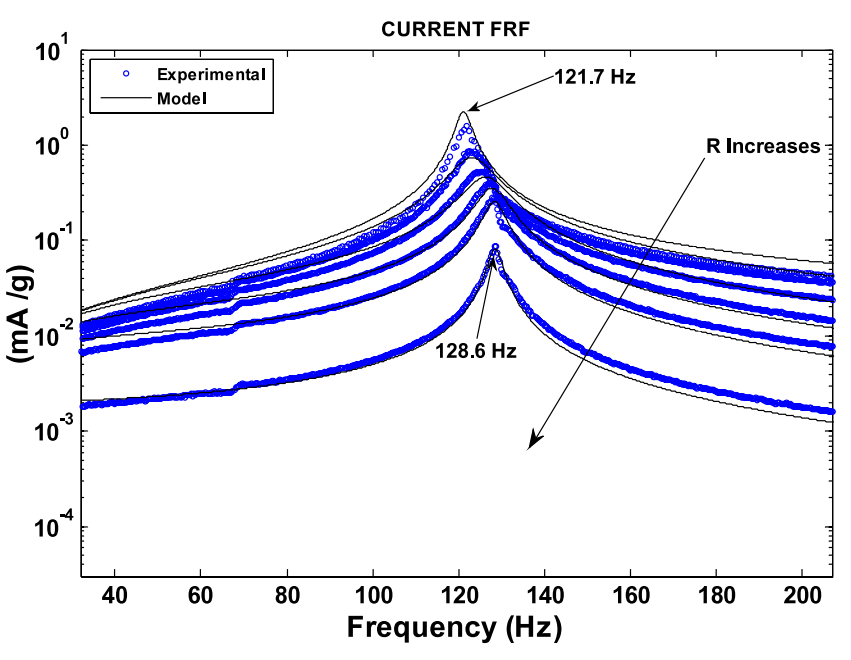

Figure 11. Current FRF at six different loads (1, 10, 25, 50, 100, $500 \mathrm{k} \Omega$ ).

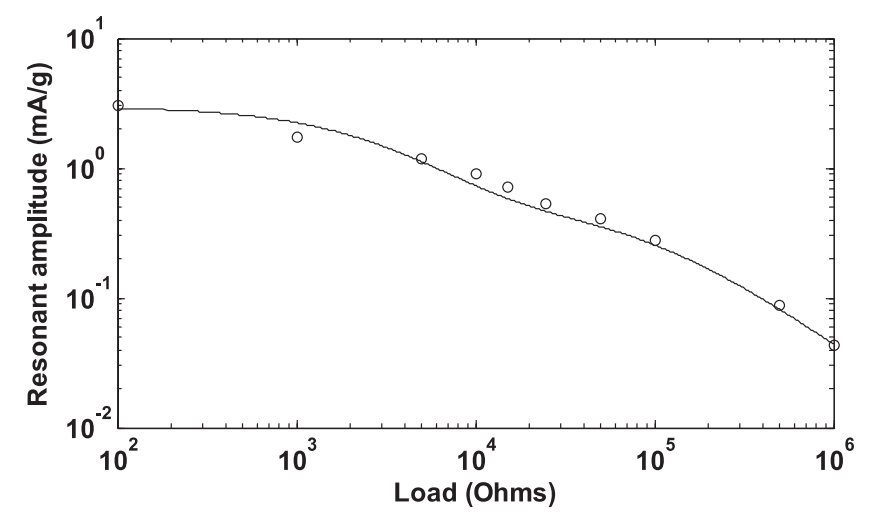

Figure 12. Variation of resonant current amplitude with electrical load (theoretical with experimental data points).

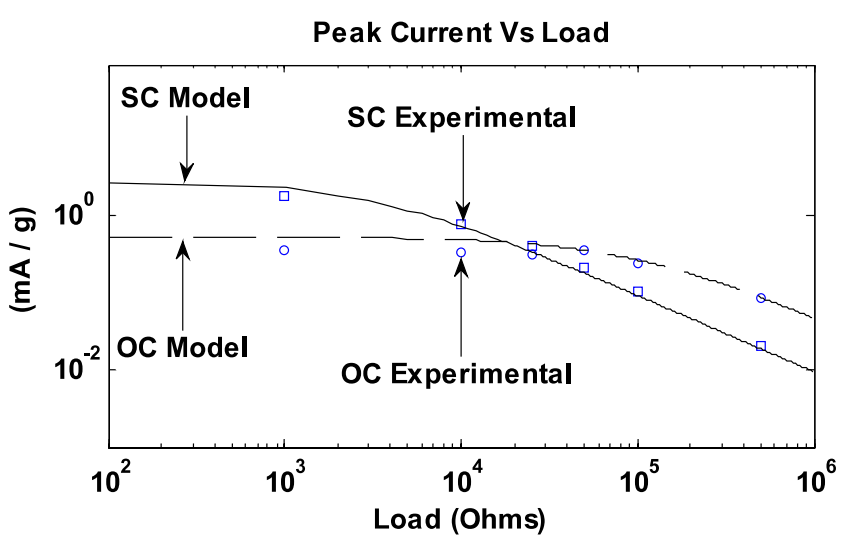

Figure 13. Current amplitude at short circuit and open circuit frequencies.

3.2.3. Power output FRF. The power FRF was defined in section 2.3 as $\left.\breve{V}(\omega)\right|^{2} / R$. This is the same as the product of the moduli of the voltage and current FRFs. Since these two FRFs have opposite monotonic variations with load, the variation of the power FRF with load will not be monotonic. 

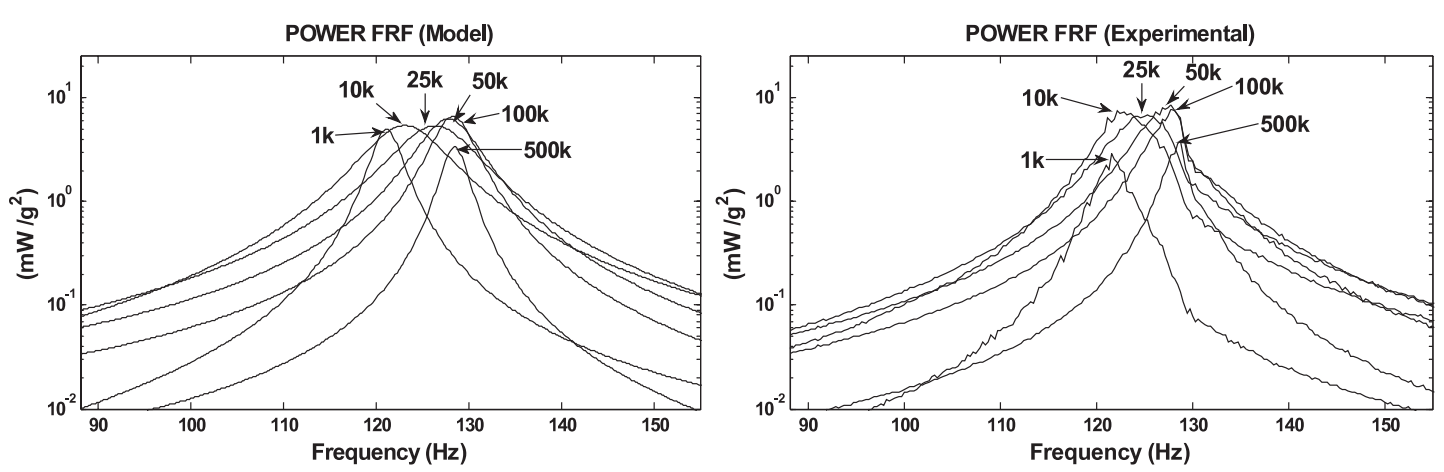

Figure 14. Power FRFs comparison: (a) theoretical; (b) experimental.

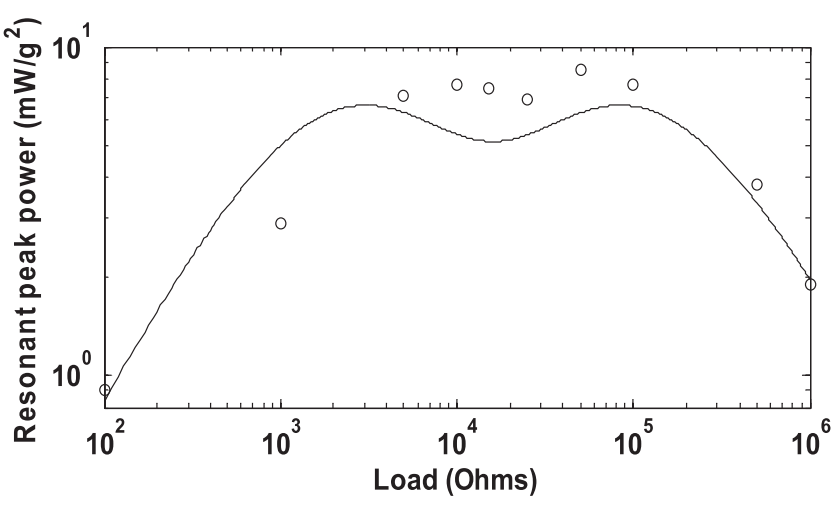

Figure 15. Variation of resonant power FRF with load (theoretical with experimental data points).

This is evident in figure 14, which shows the comparison of power FRFs obtained by the model and the experimental data for six different loads. The maxima of the power FRFs in figure 14 occur at the resonance frequencies depicted in figure 8. The power FRF values at these maxima are referred to here as 'resonant power'. Figure 15 shows the variation of the resonant power with load. The theoretical graph shows that the resonant power has turning points at loads of $3,16.2$, $85 \mathrm{k} \Omega$, with the middle load giving a local minimum point and the other two giving practically equal maxima. One should note that the horizontal axis is logarithmic. Hence, the first peak (occurring in a low resistance regime where the horizontal subdivisions represent smaller increments) would appear much sharper (i.e. thinner) when plotted on a linear horizontal (resistance) scale. The optimal resonant load of $85 \mathrm{k} \Omega$ is validated by the experimental data, which show an optimal load in the range $50-100 \mathrm{k} \Omega$. The measured data appears to show another peak occurring at a lower resistance value and the ordinates of the two measured data peaks are within $50 \%$ of each other.

The power curve in figure 15 can be obtained by squaring the resonant voltage FRF values in figure 9 and dividing by the corresponding resistance values. Hence, discrepancies between theory and experiment are amplified as a result of the squaring. However, the main reason why discrepancies appear large in figure 15 is simply because the range of the vertical axis is much narrower than that in figure 9 (the limits are 1-10 in figure 15 and $0.1-100$ in figure 9).

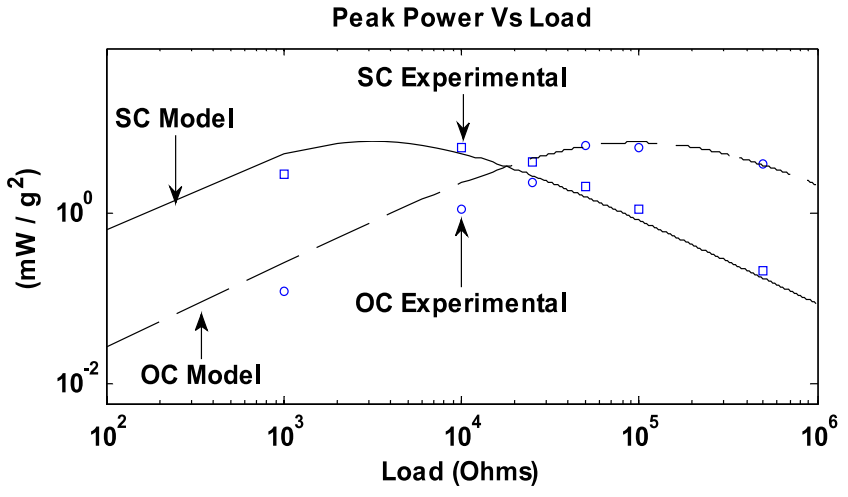

Figure 16. Power FRF at short circuit and open circuit frequencies.

Figure 16 shows the variation with load of the power FRF evaluated at two fixed frequencies: the short and open circuit resonances. It can be observed that these curves have only one turning point and that the maximum value of power is almost the same in short circuit and open circuit conditions. The optimum load is lower when the system is excited at the short circuit frequency.

3.2.4. FRFs of tip response. Figure 17 shows the theoretical and experimental results for the absolute tip displacement FRF $\beta_{\mathrm{abs}}(\omega)$ for six different loads. As previously noted, the laser measured $\beta_{\mathrm{abs}}(\omega)$, so the theoretical results were obtained by combining equations (29) and (27). It can be seen that both experiment and simulations exhibit similar behavior for the rise and fall of the resonant tip response amplitudes with load. It is important to note that changing the electrical load not only affects the amplitude of the tip but also shifts the resonance frequency of the system in the same way as it was observed in voltage, current and power FRFs. It is noted that the resonances occur at frequencies in excess of $100 \mathrm{~Hz}$, for which the $1 / \omega^{2}$ term in equation (27) is negligible. Hence, the resonances in $\beta_{\mathrm{abs}}(\omega)$ and $\beta(\omega)$ are coincident. Hence, to locate the theoretical resonance frequency at a given resistance one can differentiate the modulus of the relative displacement FRF in equation (29) and set the equation to zero. The resulting polynomial equation is given in the appendix at the end. The resonances obtained from this equation for different loads produce a graph that is virtually coincident with that of 

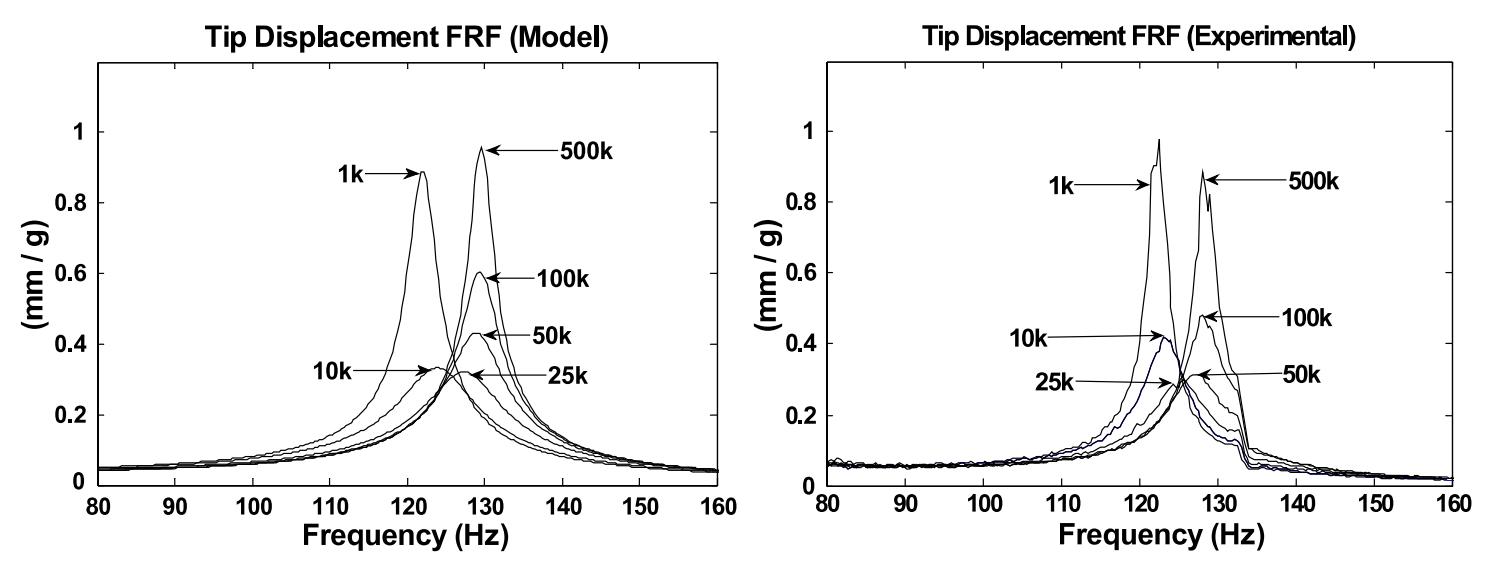

Figure 17. Absolute tip response FRFs comparison: (a) model; (b) experimental.

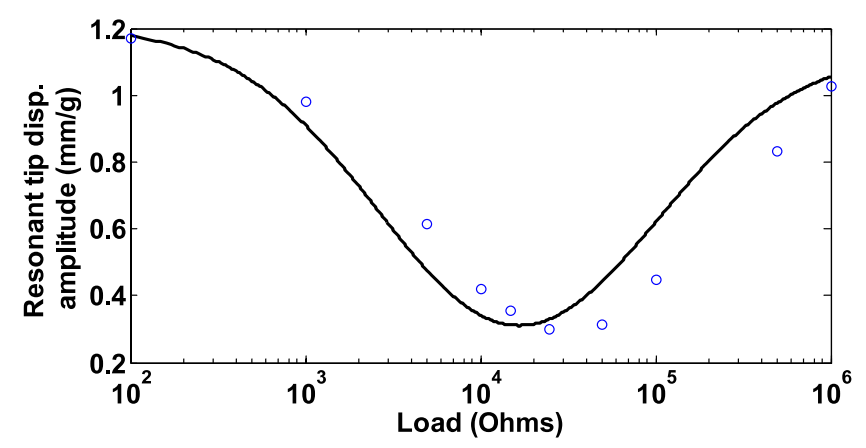

Figure 18. Variation of resonant value of absolute (or relative) tip response FRF with load (theoretical with experimental data points).

figure 8. Figure 18 shows the theoretical and experimental variation of the resonant value of $\beta_{\mathrm{abs}}(\omega)$ with electrical load. It can be observed that at the short circuit condition (when the electrical power generated is negligible) the resonant tip response is high. The resonant tip response then starts to decrease as the electrical load is increased up to a certain value. Beyond this value of electrical load, the resonant tip response starts to increase again as open circuit conditions are reached, for which the electrical power generated is negligible. It is therefore obvious that when energy is removed from the system during the energy harvesting process, additional damping is added that reduces the tip response of the beam. The fine resolution of the theoretical graph in figure 18 reveals that the resonant tip response is minimum for a theoretical load of $17 \mathrm{k} \Omega$. It is important to note that, although the electrical power generation results in additional damping, the theoretical load that gives the lowest resonant tip response in figure 18 $(17 \mathrm{k} \Omega)$, does not correspond to the theoretical load that gives the maximum resonant power output in figure $15(85 \mathrm{k} \Omega)$. In fact, the theoretical load that gives the lowest resonant tip response in figure 18 closely corresponds to the theoretical load that gives the local minimum point in the middle of figure 15 (i.e. $16 \mathrm{k} \Omega$ ). This can be explained by the fact that lowering of the tip response (i.e. reduction in deformation) tends to limit the power generated.

This feature is validated by the experimental data in figures 18 and 15: the load of $25 \mathrm{k} \Omega$ gave the lowest measured resonant tip response and a local minimum of the measured resonant power. It is interesting to note that this feature was not observed in the theoretical analysis of [21] and the theoretical and experimental analyses of [1] since resistance values were adjusted in larger steps than in the present case.

3.2.5. Evolution of Nyquist plots with load. Previous research, e.g. [1], has only considered the magnitude of the FRFs. However, the FRFs are complex-valued functions that have both magnitude (amplitude ratio) and phase information. Hence, a more thorough validation of the derived FRFs in equations (28) and (29) is achieved by studying the Nyquist plots. Figures 19 and 20 show the evolution with increasing resistance of the Nyquist plots of the voltage FRF and flexural tip velocity FRF first shown in figure 5. It is noted that the plots of both types of FRF remain approximately circular. The orientation of the circle of the voltage FRF (figure 19) relative to the origin is seen to be significantly affected by the resistance value, unlike the circle of the flexural tip velocity FRF (figure 20). The reason for this is that the numerator of the voltage FRF (equation (28)) is directly proportional to the resistance $R$. On the other hand, in the numerator of the tip deflection FRF (equation (29)), the resistance term is small compared to the other term (i.e. 2). That the theoretical and experimental Nyquist plots exhibit the same behavior with increasing resistance is a powerful validation of the model.

It is noted that, despite the reasonably good correlation between theory and prediction in figures 19 and 20, the experimental data points show deviations from the main circular pattern at some of the higher frequencies. These deviations are in the form of a minor circular outgrowth from the main circular pattern. They correspond to the kink (shoulder) at around $133 \mathrm{~Hz}$ observed mainly in the experimental tip response FRF magnitude plots in figure 17(b) and, to a lesser extent, the experimental voltage FRF plots in figure 7. It is likely that this effect is due to an unwanted torsional mode (definitely not bending) that was inadvertently excited in the experiments.

3.2.6. Limitations of theory. The authors consider that measurement error alone is not enough to account for 
$10 \mathrm{k} \Omega$ (experimental)

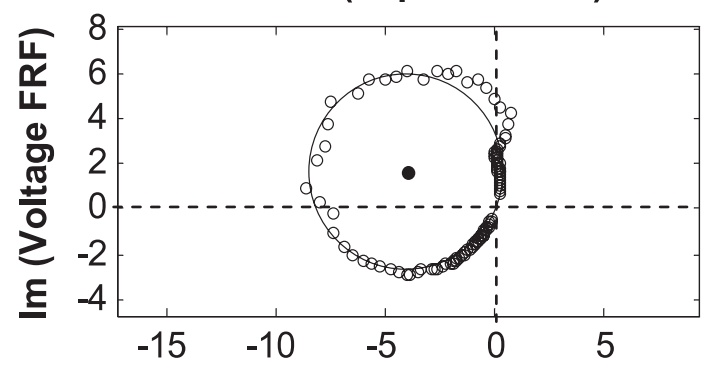

$25 \mathrm{k} \Omega$ (experimental)

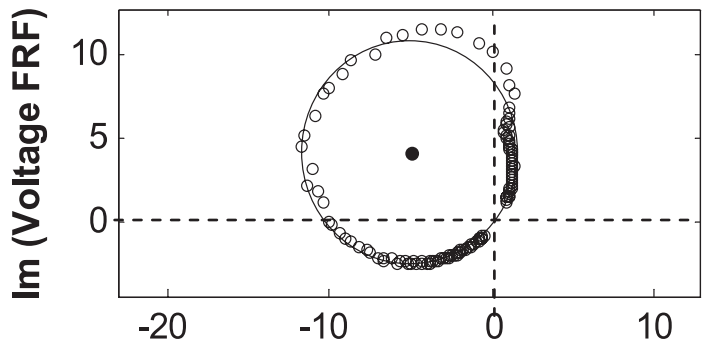

$100 \mathrm{k} \Omega$ (experimental)

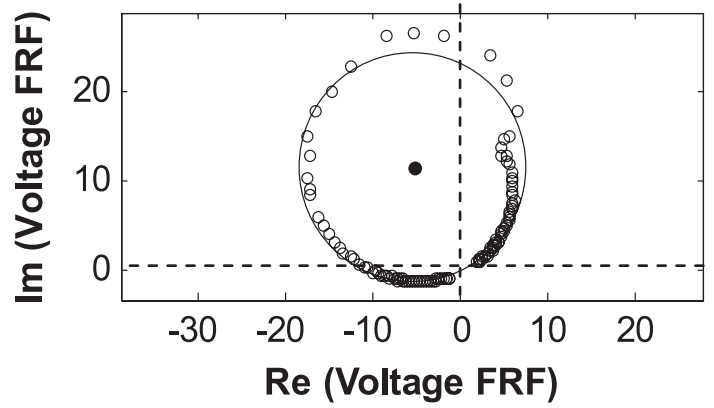

$10 \mathrm{k} \Omega$ (model)
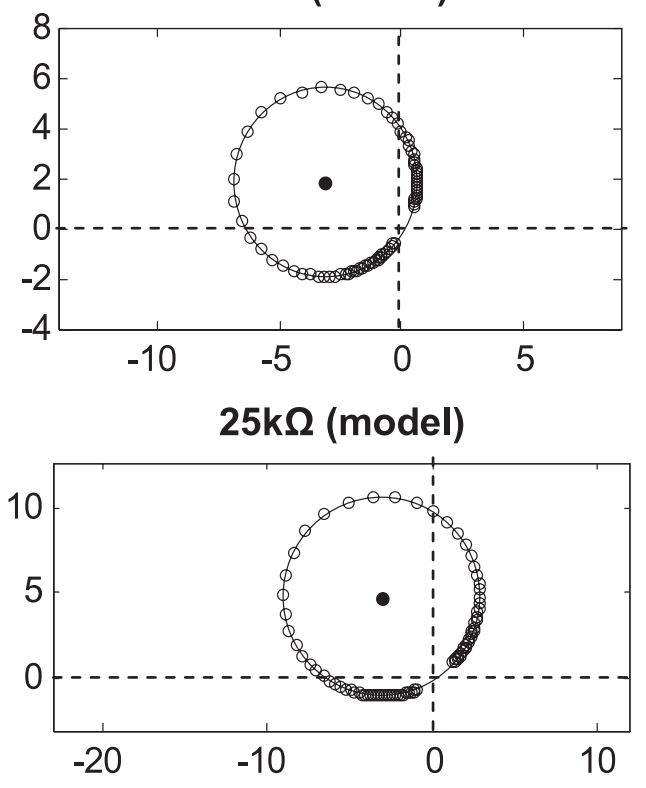

$100 \mathrm{k} \Omega$ (model)

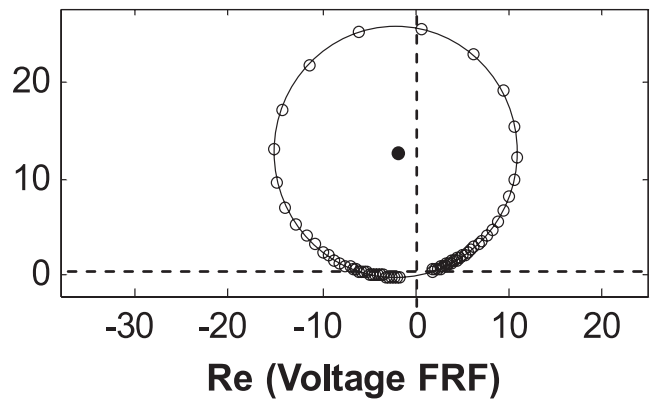

Figure 19. Nyquist plots of voltage FRF (including circle-fit through data points; $\omega$ increases clockwise from the origin).

discrepancies between theory and experiment. The following assumptions are also likely to contribute to the discrepancies.

- The electrodes that bracket the upper and lower surfaces of each piezo layer were assumed to be perfectly conductive so that a single potential could be assumed for each electrode surface.

- The bonding between the piezo layers and shim was assumed to be perfect and the impedance of the adhesive used was taken to be negligible.

It is also noted that the bimorphs were manufactured by Piezo Systems Inc., who also supplied the parameters in table 1. It may well be that deviations from these assumptions would be equivalent to an unaccounted small electrical impedance. However, its mechanism would most likely be far more complex than that of a simple additional impedance. Investigation of this possibility is beyond the scope of the present research (in which the theory is restricted to a simple resistive impedance).

\section{Conclusions}

In this paper, a distributed parameter model of a base-excited piezoelectric bimorph clamped-free beam with no tip mass was discussed and its experimental validation was performed. The experimental work successfully confirmed the accuracy of the distributed parameter modeling at a considerably higher frequency than that tested in previous research. Frequency response functions (FRFs) of the voltage, current, power and tip response were studied at different electrical loads ranging from $10^{2}$ to $10^{6} \Omega$. Theoretical and experimental graphs showing the variation with load of the resonance frequency, resonant voltage, resonant power and resonant tip response have been presented. It was shown that the electrical effect can only be regarded as a pure viscous damper at very low electrical loads. The model predicted a $6 \%$ shift in the resonance frequency of the harvester as the load was changed from the short circuit (SC) condition to the open circuit (OC) condition, and this was confirmed by the experimental results. In between the SC and OC conditions the energy harvesting effect resulted in additional damping that reduced the tip response. However, it has been revealed in this paper that the 

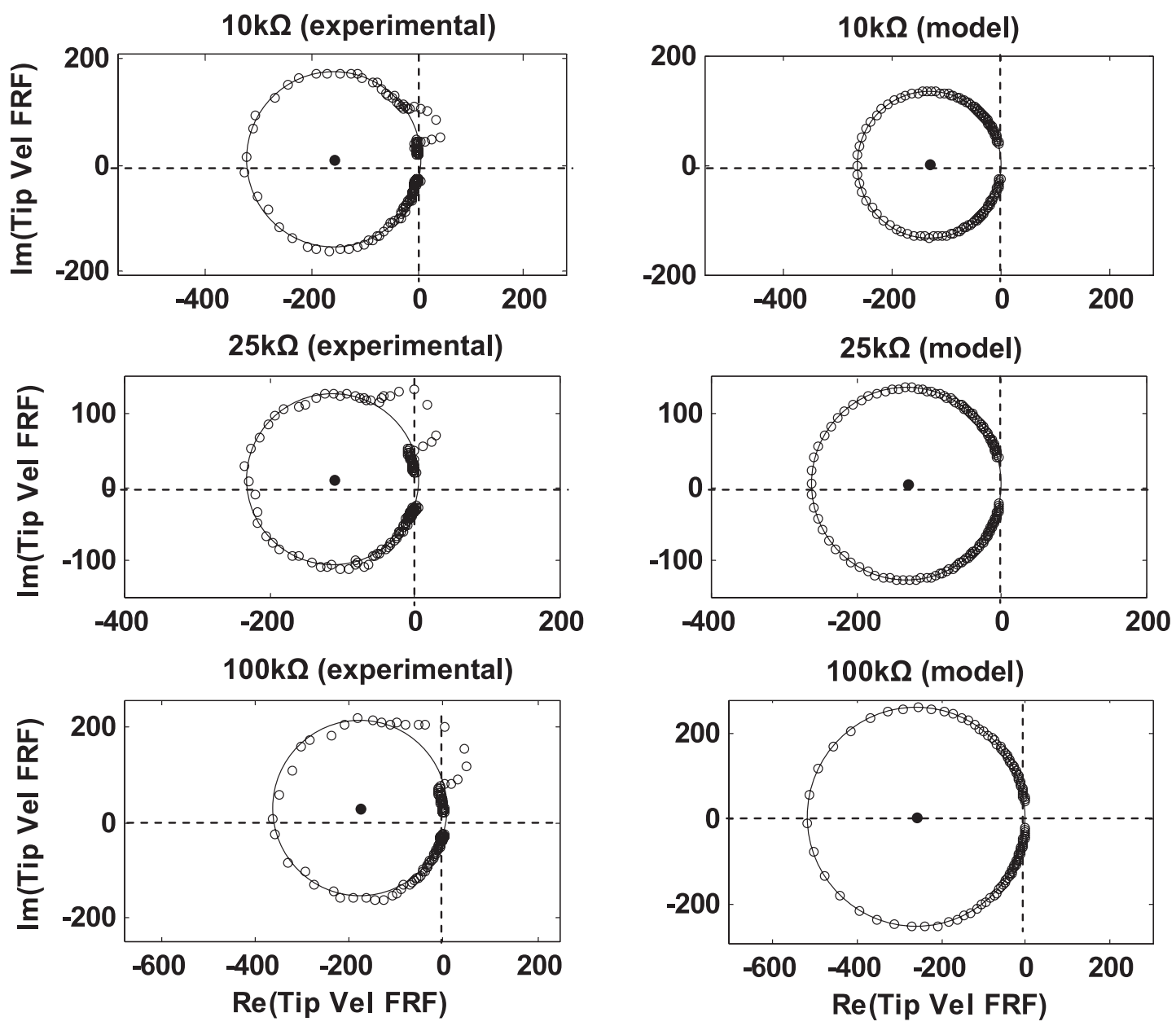

Figure 20. Nyquist plot of tip velocity FRF (including circle-fit through data points; $\omega$ increases clockwise from the origin).

conditions for maximum power generation are not the same as those for maximum mechanical damping. The load that gave maximal resonant power was much higher than the load that gave minimal tip response. The latter load was very much closer to the load that gave a minimum turning point on the resonant power versus load graph, since power generation depends on the mechanical deformation. These findings were backed by experimental evidence. Nyquist plots have been used to provide a thorough validation of the FRFs and to provide a self-validating means of estimating the mechanical damping. The modal-based theoretical analysis used in this paper will be verified for the first time against a completely different theoretical method in a forthcoming paper [32].

\section{Appendix}

The frequency that gives maximum modulus of $\left.\beta(\omega)\right|_{r}$ in equation (29) (i.e. the resonance frequency) satisfies the following cubic in $\omega^{2}$ :

$$
B_{\mathrm{a}}\left(\omega^{2}\right)^{3}+B_{\mathrm{b}}\left(\omega^{2}\right)^{2}+B_{\mathrm{c}} \omega^{2}+B_{d}=0
$$

where

$B_{\mathrm{a}}=2 K_{d}^{2} K_{\mathrm{a}}^{2}$

$$
\begin{aligned}
B_{\mathrm{b}}= & -2 K_{d}^{2} K_{\mathrm{a}} K_{\mathrm{c}}+K_{d}^{2} K_{\mathrm{a}}^{2} K_{\mathrm{b}}^{2}+4 K_{d}^{2}+3 K_{e}^{2} K_{\mathrm{a}}^{2} \\
& -2 K_{d}^{2} K_{\mathrm{a}}^{2} \omega_{r}^{2} \\
B_{\mathrm{c}}= & 8 K_{e}^{2}+2 K_{e}^{2} K_{\mathrm{a}}^{2} K_{\mathrm{b}}^{2}-4 K_{e}^{2} K_{\mathrm{a}} K_{\mathrm{c}}-4 K_{e}^{2} K_{\mathrm{a}}^{2} \omega_{r}^{2} \\
B_{d}= & 4 K_{e}^{2} K_{\mathrm{b}}^{2}-8 K_{e}^{2} \omega_{r}^{2}+K_{e}^{2} K_{\mathrm{c}}^{2}+4 K_{e}^{2} K_{\mathrm{b}} K_{\mathrm{c}} \\
& +2 K_{e}^{2} K_{\mathrm{a}} K_{\mathrm{c}} \omega_{r}^{2}+K_{e}^{2} K_{\mathrm{a}}^{2} \omega_{r}^{4}-4 K_{d}^{2} \omega_{r}^{4} \\
K_{\mathrm{a}}= & R C_{\mathrm{p}}, \\
K_{\mathrm{b}}= & 2 \xi_{r} \omega_{r}, \\
K_{\mathrm{c}} & =2 R \alpha_{r} \chi_{r}, \\
K_{e}= & R C_{\mathrm{p}} m \gamma_{r}^{u} \phi_{r}(L), \quad K_{e}=2 m \gamma_{r}^{u} \phi_{r}(L) .
\end{aligned}
$$

\section{References}

[1] Erturk A and Inman D J 2009 An experimentally validated bimorph cantilever model for piezoelectric energy harvesting from base excitations Smart Mater. Struct. 18025009

[2] Roundy S, Wright P K and Rabaey J M 2004 Energy Scavenging for Wireless Sensor Networks with Special Focus on Vibrations (Boston, MA: Kluwer Academic)

[3] Anton S R and Sodano H A 2007 A review of power harvesting using piezoelectric materials (2003-2006) Smart Mater. Struct. 16 R1-21 
[4] Priya S 2007 Advances in energy harvesting using low profile piezoelectric transducers J. Electroceram. 19 165-82

[5] Erturk A and Inman D J 2008 Mechanical considerations for modeling of vibration-based energy harvesters Proc. of the ASME Int. Design Engineering Technical Conferences and Computers and Information in Engineering Conference

[6] Erturk A and Inman D J 2008 On mechanical modeling of cantilevered piezoelectric vibration energy harvesters J. Intell. Mater. Syst. Struct. 19 1311-25

[7] Ajitsaria J, Choe S Y, Shen D and Kim D J 2007 Modeling of bimorph piezoelectric cantilever beam for voltage generation Sensors and Smart Structures Technologies for Civil, Mechanical, and Aerospace Systems 2007 (San Diego, CA: SPIE)

[8] Roundy S 2005 On the effectiveness of vibration-based energy harvesting J. Intell. Mater. Syst. Struct. 16 809-23

[9] Sodano H A, Park G and Inman D J 2004 Estimation of electric charge output for piezoelectric energy harvesting Strain $4049-58$

[10] Williams C B and Yates R B 1996 Analysis of a micro-electric generator for microsystems Sensors Actuators A 52 8-11

[11] Cornwell P J, Goethal J, Kowko J and Damianakis M 2005 Enhancing power harvesting using a tuned auxiliary structure J. Intell. Mater. Syst. Struct. 16 825-34

[12] Erturk A and Inman D J 2008 Issues in mathematical modeling of piezoelectric energy harvesters Smart Mater. Struct. 17065016

[13] Sodano H A, Lloyd J and Inman D J 2006 An experimental comparison between several active composite actuators for power generation Smart Mater. Struct. 15 1211-6

[14] Shu Y C and Lien I C 2006 Analysis of power output for piezoelectric energy harvesting systems Smart Mater. Struct. 151499

[15] Ng T-H and Liao W H 2004 Feasibility study of a self-powered piezoelectric sensor Proc. SPIE-Int. Soc. Opt. Eng. 5389 377-88

[16] Han J V J, Annette V J, Triet L, Mayaram K and Fiez T S 2004 Novel power conditioning circuits for piezoelectric micro power generators APEC: Conf. Proc.-IEEE Applied Power Electronics Conference and Exposition
[17] Ottman G K 2003 Optimized piezoelectric energy harvesting circuit using step-down converter in discontinuous conduction mode IEEE Trans. Power Electron. 18 696-703

[18] Sodano H A and Inman D J 2005 Generation and storage of electricity from power harvesting devices J. Intell. Mater. Syst. Struct. 16 67-75

[19] Sodano H A and Inman D J 2005 Comparison of piezoelectric energy harvesting devices for recharging batteries J. Intell. Mater. Syst. Struct. 16 799-807

[20] DuToit N, Wardle B L and Kim S 2005 Design considerations for MEMS-scale piezoelectric mechanical vibration energy harvesters Integr. Ferroelectr. 71 121-60

[21] Erturk A and Inman D J 2008 Distributed parameter electromechanical model for cantilevered piezoelectric energy harvesters J. Vib. Acoust. 130041002

[22] Lesieutre G A, Ottman G K and Hoffman H F 2004 Damping as a result of piezoelectric energy harvesting J. Sound Vib. 269 991-1001

[23] Timoshenko S, Young D H and Weaver W 1974 Vibration Problems in Engineering (New York: Wiley)

[24] Inman D J 2008 Engineering Vibration 3rd edn (Upper Saddle River, NJ: Pearson Prentice-Hall)

[25] Blevin R D 1984 Formulas for Natural Frequency and Mode Shape ed E Robert (Malabar, FL: Krieger)

[26] IEEE 1987 IEEE Standard on Piezoelectricity (New York: IEEE)

[27] Kim S 2002 Low power energy harvesting with piezoelectric generators Doctoral Dissertation School of Engineering, University of Pittsburgh

[28] Bendat J S and Piersol A G 1971 Random Data: Analysis and Measurement Procedures (New York: Wiley-Interscience)

[29] Kidner M R F and Brennan M J 2002 Varying the stiffness of a beam-like neutraliser under fuzzy logic control Trans. ASME, J. Vib. Acoust. 124 90-9

[30] Bonello P and Groves K H 2009 Vibration control using a beam-like adaptive tuned vibration absorber with actuator-incorporated mass-element Proc. Inst. Mech. Eng. C 223 1555-67

[31] Ewins D J 2000 Modal Testing: Theory, Practice, and Application 2nd edn (Baldock: Research Studies Press)

[32] Bonello P and Rafique S 2010 Distributed parameter modelling and analysis of a piezoelectric energy harvesting beam 10th Int. Conf. RASD 2010 (Southampton, July 2010) 